T. OTSUKI

KODAI MATH. J.

8 (1985), $375-419$

\title{
A CERTAIN PROPERTY OF GEODESICS OF THE FAMILY OF RIEMANNIAN MANIFOLDS $O_{n}^{2}$ (VII)
}

\author{
By TOMINOSUKE OTSUKI
}

\section{$\S 0 . \quad$ Introduction.}

This is exactly a continuation of Part (VI) ([16]) with the same title written by the present author which proved the following conjecture is true for $9.7 \leqq n$ $\leqq 16$. On the methods used in it, the lower bound 9.7 of this effective interval is near the crucial values from the argument in it. We shall show that this conjecture is also true for $5 \leqq n \leqq 9.7$ in the present paper by improving them and some new ideas. As the previous one we shall use the numerical data obtained by computors in the verification. We shall also use the same notation in the previous ones, Parts $(\mathrm{I}) \sim(\mathrm{VI})$.

The period $T$ of any non-trivial solution $x(t)$ of the non-linear differential equation of order 2 :

$$
n x\left(1-x^{2}\right) \frac{d^{2} x}{d t^{2}}+\left(\frac{d x}{d t}\right)^{2}+\left(1-x^{2}\right)\left(n x^{2}-1\right)=0
$$

with a constant $n>1$ such that $x^{2}+x^{\prime 2}<1$ is given by the integral:

$$
T=\sqrt{n c} \int_{x_{0}}^{x_{1}}-\overline{\sqrt{ }(n-x)} \frac{d x}{\left\{x(n-x)^{n-1}-c\right\}},
$$

where $x_{0}=n\{\min x(t)\}^{2}, \quad x_{1}=n\{\max x(t)\}^{2}, \quad 0<x_{0}<1<x_{1}<n$ and $c=x_{0}\left(n-x_{0}\right)^{n-1}=$ $x_{1}\left(n-x_{1}\right)^{n-1}$.

Conjecture $C$. The period $T$ as function of $\tau=\left(x_{1}-1\right) /(n-1)$ and $n$ is monotone decreasing with respect to $n(>2)$ for any fixed $\tau(0<\tau<1)$.

Here the author thanks heartily Professor Naoto Abe for his cooperation in the numerical computations by computors.

\section{$\S 1$. The fundamental principle to attain the purpose.}

Setting $T=\Omega(\tau, n)$, we have the formulas

$$
\frac{\partial \Omega(\tau, n)}{\partial n}=-\frac{\sqrt{c}}{2 b^{2} n \sqrt{n}} \int_{x_{0}}^{1} \frac{(1-x) \sqrt{x(n-x)^{n-1}-c} V\left(x, x_{1}\right) d x}{x^{2}(n-x)^{n}}
$$

Received March 8, 1985 
((7.4) and Proposition 3 in (III)), where $b=\sqrt{B-c}, B=(n-1)^{n-1}$ and $V\left(x, x_{1}\right)$ are defined as follows:

$$
V\left(x, x_{1}\right):=\frac{x^{2} N\left(x, x_{1}\right)}{(1-x)^{6} \sqrt{n-x}}+\frac{X^{2} N\left(X, x_{1}\right)}{(X-1)^{5} \sqrt{n-X}}
$$

where

$$
\begin{aligned}
& f_{0}(z):=(2 n-1-z) B-(n-z)^{n-1}\left\{n-z+(n-1) z^{2}\right\}, \\
& f_{1}(z):=\{4 n-1-(2 n+1) z\} B-(n-z)^{n-1}\left\{n+(2 n-1) z-(n+1) z^{2}\right\}, \\
& F_{2}(z):=-P_{2}(z) B+(n-z)^{n-1} P_{3}(z), \\
& P_{2}(z):=(2 n+1) z^{2}-2\left(2 n^{2}+5 n-4\right) z+16 n^{2}-16 n+3, \\
& P_{3}(z):=-(n-1) z^{3}+\left(2 n^{2}-7 n+8\right) z^{2}+(n-3)(4 n-1) z+3 n(2 n-1), \\
& \lambda(z):=\log (n-z)+\frac{n-1}{n-z}, \quad \phi(z):=z(n-z)^{n-1}, \\
& \tilde{\lambda}(z):=\lambda(z)-\frac{n}{n-1} \cdot \frac{(z-1)^{2}}{z(n-z)}=\log (n-z)+\frac{n z-1}{(n-1) z}, \\
& N\left(z, x_{1}\right):=(n-z) F_{2}(z)\left\{\lambda(z)-\tilde{\lambda}\left(x_{1}\right)\right\}+3(z-1)^{2} f_{0}(z)-2 n(z-1)^{3}\{B-\psi(z)\},
\end{aligned}
$$

and $X=X_{n}(x), 0<x<1<X<n$, defined by $\phi(x)=\phi(X)$.

$V\left(x, x_{1}\right)$ is increasing with respect to $x_{1}$ in $X_{n}(x) \leqq x_{1}<n$ for $0<x<1$ by Lemma 8.1 in (III). If we have $V\left(x, X_{n}(x)\right)>0$ for $0<x<1$, then we obtain $V\left(x, x_{1}\right)>0$ for $0<x<1$ and $X_{n}(x) \leqq x_{1}<n$ and so from (1.1) the inequality

$$
\frac{\partial \Omega(\tau, n)}{\partial n}<0 \text {. }
$$

We know that $V\left(x, X_{n}(x)\right)<0$ near $x=0$ when $2<n<(5+\sqrt{13}) / 4=2.15138 \cdots$ and near $x=1$ when $2<n<(1+\sqrt{13}) / 2=2.30277 \cdots$ by Lemma 8.1 in (III). But we shall show that the above argument is effective for $5 \leqq n \leqq 9.7$.

$\mathrm{W}$ have the formula (8.1) in (III) :

$$
\begin{aligned}
V(x, & X(x))=\frac{x^{2} \sqrt{n-x} F_{2}(x)\{\lambda(x)-\tilde{\lambda}(X)\}}{(1-x)^{5}}-\frac{3 x^{2} f_{0}(x)}{(x-1)^{3} \sqrt{n-x}} \\
& +\frac{2 n x^{2}\{B-\phi(x)\}}{(1-x)^{2} \sqrt{n-x}}+\frac{n}{n-1} \cdot \frac{X F_{2}(X)}{(X-1)^{3} \sqrt{n-X}}+\frac{3 X^{2} f_{0}(X)}{(X-1)^{3} \sqrt{n-X} X} \\
& -\frac{2 n X^{2}\{B-\phi(X)\}}{(X-1)^{2} \sqrt{n-X}},
\end{aligned}
$$

where $X=X(x)=X_{n}(x)$, and by Lemma 2.5 in (IV) and (2.4) and (2.5) in (VI) 


$$
\begin{gathered}
V(x, X(x))>\frac{2 n(n-X)^{2(n-1)}\{B-\phi(x)\} \sqrt{B}}{\left\{B-(X-1)^{n-1}\right\}^{2} \phi \sqrt{n B}-(X-1)^{n-1}}+\frac{2 n X \sqrt{n-X} B}{(n-1)(X-1)^{3}} H(X) \\
+\frac{x^{2} \sqrt{n-x} F_{2}(x)\{\lambda(x)-\tilde{\lambda}(X)\}}{(1-x)^{5}} \quad \text { for } 0<x<1,
\end{gathered}
$$

where

$$
H(X):=\left(\frac{n-X}{n-1}\right)^{n-1}\left\{(n-1) X^{2}+(2 n-5) X+3 n\right\}-\{8 n-5-(2 n+1) X\} .
$$

For $n>5 / 2$ we defined the constant $b=b_{n}$ by $H(b)=0,1<b<n$, and proved that

$$
\frac{2 n+10}{2 n+1}<b_{n}<2.268 \quad \text { for } n \geqq 4
$$

and $b_{n}$ is decreasing with respect to $n(\geqq 4)$ by Table 1 , Lemma 4.1 and Lemma 4.3 in (VI).

Since we have

we obtain

$$
H(X)>0 \quad \text { for } \quad b_{n}<X<n
$$

$$
V(x, X(x))>0 \quad \text { for } \quad 0<x \leqq X_{n}{ }^{-1}\left(b_{n}\right)
$$

from (1.2), when $n>5 / 2$. Therefore we shall prove the inequality:

when $n \geqq 5$.

$$
V(x, X(x))>0 \quad \text { for } \quad X_{n}{ }^{-1}\left(b_{n}\right)<x<1,
$$

In the following, we set for simplicity

$$
\begin{aligned}
U_{0}(x) & :=\frac{x^{2} \sqrt{n-x}}{(1-x)^{5}} F_{2}(x)\{\lambda(x)-\tilde{\lambda}(X(x))\}, \quad U_{1}(x):=\frac{3 x^{2} f_{0}(x)}{(x-1)^{9} \sqrt{n-x}}, \\
\text { (1.16) } \quad U_{2}(x) & :=\frac{2 n x^{2}\{B-\psi(x)\}}{(1-x)^{2} \sqrt{n-x}}, \quad U_{3}(x):=\frac{n x F_{2}(x)}{(n-1)(x-1)^{3} \sqrt{n-x}}, \\
U_{4}(x) & :=U_{3}(X(x)), \quad U_{5}(x):=U_{1}(X(x)), \quad U_{6}(x):=U_{2}(X(x)),
\end{aligned}
$$

then

$$
V(x, X(x))=U_{0}(x)-U_{1}(x)+U_{2}(x)+U_{1}(x)+U_{5}(x)-U_{8}(x) .
$$

By means of Proposition 1, Lemma 2.1 in (II) and Lemma 7.1 in (III), we see that $U_{i}(x), i=0,1,2,4,5,6$, are all positive for $0<x<1$.

\section{$\S 2$. Certain evaluations of $X_{n}{ }^{-1}(X)$.}

LEMMA 2.1. When $n>2$ and $k \geqq(n+1)^{2} /(5 n-1)$, we have

$$
x=X_{n}{ }^{-1}(X)<\left(\frac{n-X}{n-1}\right)^{n-k} \quad \text { for } \quad 1<X<n \text {. }
$$


Proof. Let $1<k<n-1$, then the above ineqnality is equivalent to

$$
\left(\frac{n-X}{n-1}\right)^{n-k}\left\{n-\left(\frac{n-X}{n-1}\right)^{n-k}\right\}^{n-1}>X(n-X)^{n-1},
$$

which can be written as

$$
\frac{1}{(n-1)^{n-k}}\left\{n-\left(\frac{n-X}{n-1}\right)^{n-k}\right\}^{n-1}>X(n-X)^{k-1} \quad \text { for } \quad 1<X<n .
$$

Both sides of (2.1) have the same value $(n-1)^{k-1}$ at $X=1$. Since we have

$$
\begin{gathered}
\left(\frac{1}{(n-1)^{n-k}}\left\{n-\left(\frac{n-X}{n-1}\right)^{n-k}\right\}^{n-1}\right)^{\prime}=\frac{n-k}{(n-1)^{n-k}}\left\{n-\left(\frac{n-X}{n-1}\right)^{n-k}\right\}^{n-2}\left(\frac{n-X}{n-1}\right)^{n-k-1} \\
\left(\frac{1}{(n-1)^{n-k}}\left\{n-\left(\frac{n-X}{n-1}\right)^{n-k}\right\}^{n-1}\right)^{\prime \prime}=\frac{n-k}{(n-1)^{n-k+1}}\left\{n-\left(\frac{n-X}{n-1}\right)^{n-k}\right\}^{n-3}\left(\frac{n-X}{n-1}\right)^{n-k-2} \\
\times\left[\left\{n^{2} \cdots(k+1) n+k-1\right\}\left(\frac{n-X}{n-1}\right)^{n-k}-n(n-k-1)\right]
\end{gathered}
$$

and

we obtain

$$
\begin{aligned}
& \left(X(n-X)^{k-1}\right)^{\prime}=(n-k X)(n-X)^{k-2}, \\
& \left(X(n-X)^{k-1}\right)^{\prime \prime}=-(k-1)(2 n-k X)(n-X)^{k-3},
\end{aligned}
$$

$$
\left.\left[\left(\frac{1}{(n-1)^{n-k}}\left\{n-\left(\frac{n-X}{n-1}\right)^{n-k}\right\}^{n-1}\right)^{\prime}\right]_{X=1}=\left[X(n-X)^{k-1}\right)^{\prime}\right]_{X=1}=(n-k)(n-1)^{k-2}
$$

and

$$
\begin{gathered}
{\left[\left(\frac{1}{(n-1)^{n-k}}\left\{n-\left(\frac{n-X}{n-1}\right)^{n-k}\right\}^{n-1}\right)^{\prime \prime}\right]_{X=1}-\left[\left(X(n-X)^{k-1}\right)^{\prime \prime}\right]_{X=1}} \\
=n(n-1)^{k-4}(k-1)(2 n-k-1)>0
\end{gathered}
$$

which implies that (2.1) holds near $X=1$.

Now, we assume that (2.1) does not hold in $1<X<n$. Then there exists a value $\zeta(1<\zeta<n)$ such that

$$
\frac{1}{(n-1)^{n-k}}\left\{n-\left(\frac{n-\zeta}{n-1}\right)^{n-k}\right\}^{n-1}=\zeta(n-\zeta)^{k-1}
$$

and

$$
\frac{n-k}{(n-1)^{n-k}}\left\{n-\left(\frac{n-\zeta}{n-1}\right)^{n-k}\right\}^{n-2}\left(\frac{n-\zeta}{n-1}\right)^{n-k-1} \leqq(n-k \zeta)(n-\zeta)^{k-2},
$$

which imply

$$
\{1+(n-k-1) \zeta\}\left(\frac{n-\zeta}{n-1}\right)^{n-k} \leqq n-k \zeta .
$$

Here, we introduce an auxiliary function 


$$
\rho_{k}(X):=\frac{n-k X}{1+(n-k-1) X} \cdot\left(\frac{n-1}{n-X}\right)^{n-k},
$$

then the above inequality becomes

$$
\rho_{k}(\zeta) \geqq 1
$$

We obtain easily

$$
\rho_{k}(1)=1, \quad \rho_{k}\left(\frac{n}{k}\right)=0, \quad \lim _{X \rightarrow n-0} \rho_{k}(X)=-\infty
$$

and

$$
\begin{aligned}
& \rho_{k}{ }^{\prime}(X) \\
= & -\frac{(n-k)\left\{n(n-2)-(n+1)(n-k-1) X+k(n-k-1) X^{2}\right\}}{(n-1)\{1+(n-k-1) X\}^{2}}\left(\frac{n-1}{n-X}\right)^{n-k+1} .
\end{aligned}
$$

From $1<k<n-1$ we have $1+(n-k-1) X>0$ for $1<X<n$. Regarding the quadratic polynomial of $X$ :

$$
n(n-2)-(n+1)(n-k-1) X+k(n-k-1) X^{2},
$$

its discriminant is

$$
\begin{aligned}
D & =(n+1)^{2}(n-k-1)^{2}-4 n(n-2) k(n-k-1) \\
& =(n-1)(n-k-1)\left\{(n+1)^{2}-(5 n-1) k\right\} .
\end{aligned}
$$

Hence, if $k \geqq(n+1)^{2} /(5 n-1)$, then it must be $D \leqq 0$, therefore

which implies

$$
\rho^{\prime}{ }_{k}(X) \leqq 0 \quad \text { for } 1 \leqq X<n \text {, }
$$

$$
\rho_{k}(X)<1 \text { for } 1<X<n .
$$

This inequality contradicts to (2.3). On the other hand we see that $(n+1)^{2}$ $/(5 n-1)<n-1$ is equivalent to $n(n-2)>0$, which holds since $n>2$. Thus, we have proved the claim of this lemma.

Q.E.D.

LEMMA 2.2. When $n>2$, let $C$ and $k$ be

$$
1<C \leqq \frac{5 n-1}{2(n+1)}
$$

and

$$
\frac{n+1+(n-1) C-\sqrt{(n-1)\left\{(n-1) C^{2}-2(n+1) C+5 n-1\right\}}}{2 C} \leqq k<n-1,
$$

then we have

$$
x=X_{n}{ }^{-1}(X)<\left(\frac{n-X}{n-1}\right)^{n-k} \quad \text { for } \quad 1<X \leqq C .
$$


Proof. First we notice that $(5 n-1) / 2(n+1)<\min (5 / 2, n)$ and the left hand side of (2.6) is the small root of the quadratic equation of $t$ :

$$
C^{2} t^{2}-C\{n+1+(n-1) C\} t+\left(n^{2}-1\right) C-n(n-2)=0 .
$$

Since we have

$$
\begin{gathered}
{\left[C^{2} t^{2}-C\{n+1+(n-1) C\} t+\left(n^{2}-1\right) C-n(n-2)\right]_{t=1}} \\
=(n-2)(C-1)(n-C)>0
\end{gathered}
$$

and

$$
\begin{gathered}
{\left[C^{2} t^{2}-C\{n+1+(n-1) C\} t+\left(n^{2}-1\right) C-n(n-2)\right]_{l=n-1}} \\
=-n(n-2)<0
\end{gathered}
$$

we obtain the inequality

$$
1<\frac{\left.n+1+(n-1) C-\sqrt{(n-1)\left\{(n-1) C^{2}\right.}-2(n+1) C+5 n-1\right\}}{2 C}<n-1 .
$$

Therefore we can use the first half of the proof of Lemma 2.1. Since $1<k<$ $n-1$, the inequality (2.1) holds near $X=1$. Assume that (2.1) does not hold in the interval $1<X \leqq C$. Then, there exists a value $\zeta(1<\zeta \leqq C)$ such that

$$
\rho_{k}(\zeta) \geqq 1 \text {. }
$$

By means of Lemma 2.1, we may suppose that $k<(n+1)^{2} /(5 n-1)$. As for the quadratic polynomial of $X$ of the right hand side of (2.4):

we have

$$
k(n-k-1) X^{2}-(n+1)(n-k-1) X+n(n-2),
$$

$$
\frac{n+1}{2 k}>\frac{5 n-1}{2(n+1)} \geqq C
$$

and

$$
\left[k(n-k-1) X^{2}-(n+1)(n-k-1) X+n(n-2)\right]_{X=1}=(n-1)^{2}-(n-k)^{2}>0 .
$$

If we have

then

$$
\begin{aligned}
& k(n-k-1) C^{2}-(n+1)(n-k-1) C+n(n-2) \\
= & -C^{2} k^{2}+C\{n+1+(n-1) C\} k-\left(n^{2}-1\right) C+n(n-2)>0
\end{aligned}
$$

$$
k(n-k-1) X^{2}-(n+1)(n-k-1) X+n(n-2)>0 \quad \text { for } \quad 1 \leqq X \leqq C .
$$

From the property of the quadratic polynomial of $t$ :

$$
C^{2} t^{2}-C\{n+1+(n-1) C\} t+\left(n^{2}-1\right) C-n(n-2)
$$

stated in the begining, we see that the above fact holds from (2.6). Thus, we see that $\rho_{k}(X)$ is decreasing in $1<X \leqq C$, and hence

$$
\rho_{k}(X)<1 \quad \text { for } 1<X \leqq C,
$$


which contradicts to $\rho_{k}(\zeta) \geqq 1$.

Q.E.D.

Here, we set

$$
k_{n}(C):=\frac{n+1+(n-1) C-\sqrt{(n-1)\left\{(n-1) C^{2}-2(n+1) C+5 n-1\right\}}}{2 C},
$$

then

$$
n-k_{n}(C)=\frac{(n+1)(C-1)+\sqrt{(n-1)\left\{(n-1) C^{2}-2(n+1) C+5 n-1\right\}}}{2 C} .
$$

LEMMA 2.3. (i) $k_{n}$ (C) is increasing with respect to $C$ in $1<C \leqq(5 n-1) /(2(n+1))$, with $n>2$, and $k_{n}(1)=1$ and $k_{n}((5 n-1) /(2(n+1)))=(n+1)^{2} /(5 n-1)$.

(ii) $k_{n}(C)$ and $n-k_{n}(C)$ are increasing with respect to $n(\geqq 2.5)$, with $1<C$ $\leqq(5 n-1) /(2(n+1))$.

Proof. (i) From (2.8) we obtain

$$
\begin{aligned}
& \frac{\partial k_{n}(C)}{\partial C}=\frac{1}{2 C^{2} \sqrt{(n-1)\left\{(n-1) C^{2}-2(n+1) C+5 n-1\right\}}} \\
& \times\left[(n-1)\{5 n-1-(n+1) C\}-(n+1) \sqrt{(n-1)\left\{(n-1) C^{2}-2(n+1) C+5 n-1\right\}}\right] .
\end{aligned}
$$

Since we have

$$
5 n-1-(n+1) C \geqq 5 n-1-\frac{5 n-1}{2}=\frac{5 n-1}{2}>0
$$

and

$$
\begin{aligned}
& (n-1)^{2}\{5 n-1-(n+1) C\}^{2}-(n+1)^{2}(n-1)\left\{(n-1) C^{2}-2(n+1) C+5 n-1\right\} \\
= & 4 n(n-1)(n-2)\{5 n-1-2(n+1) C\}>0,
\end{aligned}
$$

we obtain

$$
\frac{\partial k_{n}(C)}{\partial C}>0 \quad \text { for } \quad 1<C<\frac{5 n-1}{2(n+1)}, \quad \text { with } n>2 .
$$

(ii) Differentiating the numerator of (2.8) with respect to $n$, we obtain

$$
\frac{(C+1) \sqrt{(n-1)\left\{(n-1) C^{2}-2(n+1) C+5 n-1\right\}}-\left\{(n-1) C^{2}-2 n C+5 n-3\right\}}{\sqrt{(n-1)\left\{(n-1) C^{2}-2(n+1) C+5 n-1\right\}}}
$$

and

$$
\begin{aligned}
& (C+1)^{2}(n-1)\left\{(n-1) C^{2}-2(n+1) C+5 n-1\right\}-\left\{(n-1) C^{2}-2 n C+5 n-3\right\}^{2} \\
= & 4(C-1)\left\{(n-1)^{2} C^{2}-\left(2 n^{2}-1\right) C+5 n^{2}-6 n+2\right\}>0 \quad \text { for } n \geqq 2.5,
\end{aligned}
$$

because $C>1$ and

$$
\left(2 n^{2}-1\right)^{2}-4(n-1)^{2}\left(5 n^{2}-6 n+2\right)=-\left(16 n^{4}-64 n^{3}+80 n^{2}-40 n+7\right)<0
$$

for $n \geqq 2.5$.

The claim on $n-k_{n}(C)$ is evident from the above computation. Q.E.D.

LEMMA 2.4. When $3<n \leqq 10$, we have 


$$
x=X_{n}{ }^{-1}(X)<\left(\frac{n-X}{n-1}\right)^{n-2} \quad \text { for } \quad 1<X \leqq b_{n} .
$$

Proof. By the same argument used in the proof of Lemma 2.1, it is suffcient to prove that

$$
n(n-2)-(n+1)(n-3) X+2(n-3) X^{2} \geqq 0 \quad \text { for } \quad 1<X \leqq b_{n} .
$$

The discriminant of the quadratic polynomial of $X$ is

$$
D=(n-1)(n-3)\left(n^{2}-8 n+3\right),
$$

which is non positive for $3<n \leqq 4+\sqrt{13}=7.6055 \cdots$. Therefore the claim is true for $3<n \leqq 4+\sqrt{13}$.

Now, let assume $n>4+\sqrt{ } 13$, then the polynomial is positive for

$$
1 \leqq X<\frac{1}{4}\left(n+1-\sqrt{\frac{(n-1)\left(n^{2}-8 n+3\right)}{n-3}}\right),
$$

and $(n+1) / 4<(n / 3)$ and

$$
\left[n(n-2)-(n+1)(n-3) X+2(n-3) X^{2}\right]_{X=n / 3}=-\frac{1}{9} n\left(n^{2}-n+9\right)<0 .
$$

Therefore, if

$$
\rho_{2}\left(\frac{n+1}{4}\right)=\frac{2}{n-1}\left(\frac{4(n-1)}{3 n-1}\right)^{n-2}<1
$$

and $b_{n} \leqq(n+1) / 4$, then the claim is true for this $n$.

We can prove easily that $1 /(n-1)(4(n-1) /(3 n-1))^{n-2}$ is increasing with respect to $n$ for $n>5.2$. Hence, we have for $4+\sqrt{13}<n \leqq 8.5$

$$
\rho_{2}\left(\frac{n+1}{4}\right) \leqq \rho_{2}\left(\frac{9.5}{4}\right)=\frac{2}{7.5}\left(\frac{4 \times 7.5}{24.5}\right)^{6.5}=0.9466 \cdots<1
$$

and by means of Lemma 4.3 in (VI) we have

$$
b_{n}<b_{7}=1.7949 \cdots<2<\frac{n+1}{4} .
$$

(Here we notice that $\rho_{2}((8.6+1) / 4)=1.00897 \cdots>1$.)

Next, let assume $n>8.5$. We obtain

$$
\rho_{2}\left(\frac{n}{5}\right)=\frac{3 n}{n^{2}-3 n+5}\left(\frac{5(n-1)}{4 n}\right)^{n-2}
$$

and we can prove that this is increasing with respect to $n$ for $n \geqq 8$. Hence, we have for $8.5 \leqq n \leqq 9.5$

and

$$
\rho_{2}\left(\frac{n}{5}\right) \leqq \rho_{2}(1.9)=\frac{3 \times 9.5}{66.75}\left(\frac{5 \times 8.5}{4 \times 9.5}\right)^{7.5}=0.98840 \cdots<1
$$




$$
b_{n} \leqq b_{8.5}=1.6654 \cdots<\frac{8.5}{5}=1.7 \leqq \frac{n}{5} \quad\left(<\frac{n}{3}\right) .
$$

Therefore, the claim is true for $8.5<n \leqq 9.5$.

Finally, let assume $n>9.5$, then we obtain

$$
\rho_{2}\left(\frac{n}{5.5}\right)=\frac{7 n}{2 n^{2}-6 n+11}\left(\frac{11(n-1)}{9 n}\right)^{n-2}
$$

and we can prove that this is increasing with respect to $n$ for $n \geqq 9$. Hence, we have for $9.5 \leqq n \leqq 10$

and

$$
\rho_{2}\left(\frac{n}{5.5}\right)=\rho_{2}\left(\frac{10}{5.5}\right)=\frac{70}{151}\left(\frac{11 \times 9}{9 \times 10}\right)^{8}=0.99371 \cdots<1
$$

$$
b_{n} \leqq b_{9.5}=1.5998 \cdots<\frac{9.5}{5.5}=\frac{19}{11}=1 . \dot{7} \dot{2} \leqq \frac{n}{5.5} .
$$

Therefore, the claim is also true for $9.5<n \leqq 10$.

Q.E.D.

LEMMA 2.5. (i) When $3<n \leqq 10$, we have

$$
\frac{X-1}{1-x}<e_{n-2}=\left(\frac{n-1}{n-2}\right)^{n-2} \quad \text { for } \quad 1<X \leqq \min \left(2, b_{n}\right) \text {. }
$$

(ii) When $4 \leqq n \leqq 4.5$, we have

$$
\frac{X-1}{1-x}<\left(\frac{n-1}{n-1.225}\right)^{n-1.169} \quad \text { for } 1<X \leqq 1.225 \text {. }
$$

(iii) When $4.5 \leqq n \leqq 5$, we have

$$
\frac{X-1}{1-x}<\left(\frac{n-1}{n-1.2}\right)^{n-1.165} \quad \text { for } \quad 1<X \leqq 1.163 .
$$

Proof. (i) By means of Lemma 2.4 and the means value theorem, for $1<X \leqq \min \left(2, b_{n}\right)$ we have

$$
\frac{X-1}{1-x}>\frac{X-1}{1-\left(\frac{n-X}{n-1}\right)^{n-2}}<\frac{n-1}{n-2} \cdot \frac{1}{\left(\frac{n-X}{n-1}\right)^{n-3}} \leqq\left(\frac{n-1}{n-2}\right)^{n-2}=e_{n-2} .
$$

(ii) and (iii) By means of Lemma 2.2 , for $1<C \leqq(5 n-1) /(2(n+1)), k=k_{n}(C)$, $1<X \leqq C$, we have

$$
\frac{X-1}{1-x}<\frac{X-1}{1-\left(\frac{n-X}{1-n}\right)^{n-k}}<\frac{n-1}{n-k} \cdot \frac{1}{\left(\frac{n-X}{n-1}\right)^{n-k-1}} \leqq \frac{(n-1)^{n-k}}{(n-k)(n-C)^{n-k-1}} .
$$

When $4 \leqq n \leqq 4.5$, we have $(5 n-1) / 2(n+1) \geqq(19 / 10)$. For $1<C \leqq 1.225$, we obtain by Lemma 2.3

$$
k_{n}(C) \leqq k_{4.5}(1.225)=1.21248 \cdots<1.213 .
$$


Hence we obtain

$$
\frac{(n-1)^{n-k}}{(n-k)(n-C)^{n-k-1}}<\left(\frac{n-1}{n-1.225}\right)^{n-k_{n}(C)}
$$

and

$$
k_{n}(1.225) \geqq k_{4}(1.225)=1.16940 \cdots>1.169 \text {. }
$$
have

When $4.5 \leqq n \leqq 5$, we have $(5 n-1) /(2(n+1)) \geqq 43 / 22$. For $1<C \leqq 1.163$, we

Hence we obtain

$$
k_{n}(C) \leqq k_{5}(1.163)=1.19931 \cdots<1.2 \text {. }
$$

$$
\frac{(n-1)^{n-k}}{(n-k)(n-C)^{n-k-1}} \leqq\left(\frac{n-1}{n-1.2}\right)^{n-k_{n}(C)} \text {. }
$$

and

$$
k_{n}(1.163) \geqq k_{4.5}(1.163)=1.16581 \cdots>1.165 \text {. }
$$

Q.E.D.

\section{§3. Some evaluations related with $U_{i}(x)$.}

As for $F_{2}(x)$ defined by (1.5), we have the formulas

$$
F_{2}^{\prime}(x)=-2 B\left\{(2 n+1) x-\left(2 n^{2}+5 n-4\right)\right\}-(n-x)^{n-2} Q_{3}(x),
$$

where

$$
Q_{3}(x):=2 n^{2}(n+2)+n(n-13) x+2\left(n^{3}-n^{2}-n+4\right) x^{2}-(n-1)(n+2) x^{3}
$$

by (3.2), (3.3) and (3.5) in (II), and

and

$$
\begin{aligned}
F_{2}{ }^{\prime \prime}(x)= & -2 B(2 n+1)-(n-x)^{n-3}\left\{(n-x) Q_{3}{ }^{\prime}(x)-(n-2) Q_{3}(x)\right\} \\
= & -2 B(2 n+1)-(n-x)^{n-3}\left\{-n^{2}\left(2 n^{2}-n+5\right)+n\left(4 n^{3}-5 n^{2}+10 n+3\right) x\right. \\
& \left.-n(n+1)\left(2 n^{2}-n+2\right) x^{2}+(n-1)(n+1)(n+2) x^{3}\right\},
\end{aligned}
$$

$$
F_{2}^{\prime \prime \prime}(x)=-n(n-1)(n-x)^{n-4} \tilde{Q}(x),
$$

where

and

$$
\begin{aligned}
\tilde{Q}_{3}(x):= & 6 n\left(n^{2}-n+2\right)-\left(8 n^{3}-3 n^{2}+19 n+6\right) x+2(n+1)\left(n^{2}+n+4\right) x^{2} \\
& -(n+1)(n+2) x^{3}
\end{aligned}
$$

$$
F_{2}{ }^{(4)}(x)=-n(n-1)(n-x)^{n-5} \tilde{R}_{3}(x),
$$

where

$$
\begin{aligned}
\tilde{R}_{3}(x):= & -n\left(14 n^{3}-33 n^{2}+55 n-42\right)+\left(12 n^{4}-19 n^{3}+48 n^{2}-35 n-18\right) x \\
& -\left(2 n^{4}+3 n^{3}+11 n^{2}-6 n-16\right) x^{2}+(n-1)(n+1)(n+2) x^{3} .
\end{aligned}
$$

LEMMA 3.1. When $n>2, F_{8}(x)$ is decreasing in $0<x<1$.

Proof. We get from (3.5) 
and

$$
\tilde{Q}_{3}{ }^{\prime}(x)=-\left(8 n^{3}-3 n^{2}+19 n+6\right)+4(n+1)\left(n^{2}+n+4\right) x-3(n+1)(n+2) x^{2}
$$

Since we have

$$
1<\frac{2\left(n^{2}+n+4\right)}{3(n+2)}<n \quad \text { for } n>2
$$

$$
\begin{aligned}
& \tilde{Q}_{3}{ }^{\prime}(0)=-\left(8 n^{3}-3 n^{2}+19 n+6\right)<0, \\
& \tilde{Q}_{3}{ }^{\prime}(1)=-4(n-1)\left(n^{2}-n+1\right)<0,
\end{aligned}
$$

we obtain

$$
\tilde{Q}_{3}{ }^{\prime}(x)<0 \quad \text { for } \quad 0 \leqq x \leqq 1 \text {. }
$$

Since we have

$$
\tilde{Q}_{3}(0)=6 n\left(n^{2}-n+2\right)>0 \text { and } \tilde{Q}_{3}(1)=0 \text {, }
$$

we obtain

$$
\tilde{Q}_{3}(x)>0 \quad \text { for } \quad 0 \leqq x<1
$$

and so

$$
F_{2}^{\prime \prime \prime}(x)<0 \text { for } 0 \leqq x<1 \text {. }
$$

Hence $F_{2}^{\prime \prime}(x)$ is decreasing in $0 \leqq x<1$. Now, we have

$$
\begin{aligned}
F_{2}{ }^{\prime \prime}(0) & =-2 B(2 n+1)+n^{n-1}\left(2 n^{2}-n+5\right) \\
& =(n-1)^{n-1}\left\{e_{n-1}\left(2 n^{2}-n+5\right)-2(2 n+1)\right\},
\end{aligned}
$$

where $e_{n-1}=(1+(1 / n-1))^{n-1}$. When $n>2$, we have

$$
e_{n-1}\left(2 n^{2}-n+5\right)-2(2 n+1)>2\left(2 n^{2}-n+5\right)-2(2 n+1)=2\left(2 n^{2}-3 n+4\right)>0,
$$

and hence

$$
F_{2}^{\prime \prime}(0)>0 \text {. }
$$

Since we see easily that $F_{2}^{\prime \prime}(1)=0$, it must be

$$
F_{2}^{\prime \prime}(x)>0 \text { for } 0 \leqq x<1 .
$$

Hence $F_{2}^{\prime}(x)$ is increasing in $0 \leqq x<1$. We can prove easily that $F_{2}^{\prime}(1)=0$. Hence it must be

$$
F_{2}{ }^{\prime}(x)<0 \quad \text { for } 0 \leqq x<1,
$$

which implies the claim of this lemma.

Q.E.D.

LEMMA 3.2. $x^{2} \sqrt{n-x} /(1-x)^{5}$ is increasing with respect to $x(0<x<1)$, when $n>1$.

Proof. We have easily

$$
\frac{d}{d x} \cdot \frac{x^{2} \sqrt{n-x}}{(1-x)^{5}}=\frac{x\left\{4 n+(6 n-5) x-5 x^{2}\right\}}{2(1-x)^{6} \sqrt{n-x}}>0 \text { for } 0<x<1,
$$

when $n>1$.

Regarding Lemma 2.1 we have

Q.E.D. 


$$
n-\frac{(n+1)^{2}}{5 n-1}=\frac{(n-1)(4 n+1)}{5 n-1},
$$

and so here we introduce two auxiliary functions as

$$
\begin{aligned}
& y=y(n, X):=\left(\frac{n-X}{n-1}\right)^{(n-1)(4 n+1) /(5 n-1)}, \\
& w=w(n, X):=\left(\frac{n-X}{n-1}\right)^{n-1} .
\end{aligned}
$$

When $n>2$, we have by Lemma 2.1 and Lemma 2.1 in (IV), respectively, the inequalities

$$
w(n, X)<x=X_{n}{ }^{-1}(X)<y(n, X) \quad \text { for } \quad 1<X<n .
$$

LEMMA 3.3. When $n>2$, we have

$$
\begin{aligned}
& \frac{U_{0}(x)}{B}>\frac{w^{2} \sqrt{n-w}}{(1-w)^{5}}\left\{-P_{2}(y)+\left(\frac{n-y}{n-1}\right)^{n-1} P_{3}(y)\right\} \\
& \quad \times\left\{\log \frac{n-y}{n-X}+\frac{n X y-(2 n-1) X+n-y}{(n-1) X(n-y)}\right\} \text { for } 0<x<1 .
\end{aligned}
$$

Proof. By means of (1.6), Lemma 3.1, Lemma 3.2, (3.10) and the fact that $\lambda(x)$ is decreasing in $0<x<1$, and $\tilde{\lambda}(X)$ is also decreasing in $1<X<n$, and we have

$$
\begin{aligned}
\lambda(x)-\tilde{\lambda}(X)>\lambda(y)-\tilde{\lambda}(X) & =\log (n-y)+\frac{n-1}{n-y}-\log (n-X)-\frac{n X-1}{(n-1) X} \\
& =\log \frac{n-y}{n-X}+\frac{n X y-(2 n-1) X+n-y}{(n-1) X(n-y)},
\end{aligned}
$$

we obtain immediately (3.11).

LEMMA 3.4. When $n>1, f_{0}(x)$ is negative in $0<x<1$ and it is increasing there and $f_{0}(1)=0$.

Proof. We get from (1.3)

and hence

$$
\begin{aligned}
& f_{0}^{\prime}(x)=-B+(n-x)^{n-2}\left\{\left(n^{2}-1\right) x^{2}-n(2 n-1) x+n^{2}\right\}, \\
& f_{0}^{\prime \prime}(x)=-n(n-1)(x-1)\{(n+1) x-3 n\}(n-x)^{n-3},
\end{aligned}
$$

$$
f_{0}^{\prime \prime}(x)<0 \text { for } 0<x<1 \text {, }
$$

therefore $f_{0}{ }^{\prime}(x)$ is decreasing there and $f_{0}{ }^{\prime}(1)=0$. Hence, we see that $f_{0}{ }^{\prime}(x)>0$ for $0<x<1$ and so $f_{0}(x)$ is increasing in $0<x<1$. Since $f_{0}(1)=0$, we obtain that $f_{0}(x)<0$ for $0<x<1$.

Q.E.D.

LEMMA 3.5. When $n>2$, we have

$$
-\frac{U_{1}(x)}{B}>\frac{3 y^{2}}{(1-y)^{3} \sqrt{ } n-y}\left[2 n-1-w-\left(\frac{n-w}{n-1}\right)^{n-1}\left\{n-w+(n-1) w^{2}\right\}\right] .
$$


Proof. We obtain easily this inequality from (1.16), Lemma 3.4 and (3.10).

We can easily prove the following

Q.E.D.

LEMMA 3.6. When $n>2$, we have

$$
\frac{U_{2}(x)}{B}>\frac{2 n w^{2}(1-X w)}{(1-w)^{2} \sqrt{ } n-w} \quad \text { for } \quad 0<x<1 .
$$

Since we can represent $U_{4}(x), U_{5}(x)$ and $U_{6}(x)$ as

$$
\left\{\begin{array}{l}
\frac{U_{4}(x)}{B}=\frac{n X}{(n-1)(X-1)^{3} \sqrt{n-X}} \cdot\left\{-P_{2}(X)+w P_{3}(X)\right\}, \\
\frac{U_{5}(x)}{B}=\frac{3 X^{2}}{(X-1)^{3} \sqrt{n-X}} \cdot\left[(2 n-1-X)-w\left\{n-X+(n-1) X^{2}\right\}\right], \\
\frac{U_{6}(x)}{B}=\frac{2 n X^{2}(1-X w)}{(X-1)^{2} \sqrt{n-X}} \quad \text { with } \quad X=X_{n}(x),
\end{array}\right.
$$

when $n>2$, we obtain from (3.11), (3.12), (3.13) and (3.14) the following inequality

$$
\begin{aligned}
& \frac{V(x, X(x))}{B}>\frac{w^{2} \sqrt{n-w}}{(1-w)^{5}}\left\{-P_{2}(y)+\left(\frac{n-y}{n-1}\right)^{n-1} P_{3}(y)\right\} \\
& \quad \times\left\{\log \frac{n-y}{n-X}+\frac{n X y-(2 n-1) X+n-y}{(n-1) X(n-y)}\right\} \\
& +\frac{3 y^{2}}{(1-y)^{3} \sqrt{n-y}}\left[2 n-1-w-\left(\frac{n-w}{n-1}\right)^{n-1}\left\{n-w+(n-1) w^{2}\right\}\right] \\
& +\frac{2 n w^{2}(1-X w)}{(1-w)^{2} \sqrt{n}=\frac{w}{-w}}+\frac{n X}{(n-1)(X-1)^{3} \sqrt{n-X}}\left\{-P_{2}(X)+w P_{3}(X)\right\} \\
& +\frac{3 X^{2}}{(X-1)^{3} \sqrt{n-X}}\left[2 n-1-X-w\left\{n-X+(n-1) X^{2}\right\}\right]-\frac{2 n X^{2}(1-X w)}{(X-1)^{2} \sqrt{n-X}}
\end{aligned}
$$

for $0<x<1$. Arranging the right hand side of this inequality, we obtain a fundamental formula for our purpose.

PROPOSITION 1. When $n>2$, we have

$$
V(x, X(x))>(n-1)^{n-1} K(n, X(x)) \quad \text { for } 0<x<1 \text {, }
$$

where

$$
\begin{aligned}
K(n, X): & =\frac{w^{2} \sqrt{n-\bar{w}}}{(1-w)^{5}} \cdot\left[\left\{-P_{2}(y)+\left(\frac{n-y}{n-1}\right)^{n-1} P_{3}(y)\right\}\right. \\
& \left.\times\left\{\log \frac{n-y}{n-X}+\frac{n X y-(2 n-1) X+n-y}{(n-1) X(n-y)}\right\}+\frac{2 n(1-w)^{3}(1-X w)}{n-w}\right] \\
& +\frac{3 y^{2}}{(1-y)^{3} \sqrt{n-y}} \cdot\left[2 n-1-w-\left(\frac{n-w}{n-1}\right)^{n-1}\left\{n-w+(n-1) w^{2}\right\}\right]
\end{aligned}
$$




$$
-\frac{X}{(n-1)(X-1)^{3} \sqrt{n}-X} \cdot\left[Q_{2}(X)+w Q_{3}(X)\right],
$$

$$
\begin{aligned}
Q_{2}(z):= & \left(4 n^{2}+2 n-3\right) z^{2}-\left(4 n^{3}+18 n^{2}-19 n+3\right) z+n(4 n-1)(4 n-3), \\
Q_{3}(z):= & (n-1)(2 n-3) z^{3}-\left(2 n^{3}-9 n^{2}+13 n-3\right) z^{2}-2 n\left(2 n^{2}-8 n+3\right) z \\
& -3 n^{2}(2 n-1),
\end{aligned}
$$

and $w=w(n, X), y=y(n, X)$ by (3.8), (3.9).

Proof. We show only the computation related with the last term in (3.16). In fact, from the last three terms in the inequality described above we have

$$
\begin{aligned}
Q_{2}(X)= & n P_{2}(X)-3(n-1) X(2 n-1-X)+2 n(n-1) X(X-1) \\
= & n(2 n+1) X^{2}-2 n\left(2 n^{2}+5 n-4\right) X+n(4 n-1)(4 n-3) \\
& +3(n-1) X^{2}-3\left(2 n^{2}-3 n+1\right) X+2 n(n-1) X^{2}-2 n(n-1) X \\
= & \left(4 n^{2}+2 n-3\right) X^{2}-\left(4 n^{3}+18 n^{2}-19 n+3\right) X+n(4 n-1)(4 n-3) \\
Q_{3}(X)= & -n P_{3}(X)+3(n-1) X\left\{n-X+(n-1) X^{2}\right\}-2 n(n-1) X^{2}(X-1) \\
= & n(n-1) X^{3}-n\left(2 n^{2}-7 n+8\right) X^{2}-n(n-3)(4 n-1) X-3 n^{2}(2 n-1) \\
& +3(n-1)^{2} X^{3}-3(n-1) X^{2}+3 n(n-1) X-2 n(n-1) X^{3}+2 n(n-1) X^{2} \\
= & (n-1)(2 n-3) X^{3}-\left(2 n^{3}-9 n^{2}+13 n-3\right) X^{2}-2 n\left(2 n^{2}-8 n+3\right) X-3 n^{2}(2 n-1) .
\end{aligned}
$$

In the following, we want to show that

Q.E.D.

$$
V(x, X(x))>0 \quad \text { for } \quad X_{n}^{-1}\left(b_{n}\right)<x<1
$$

by a finite number of evaluations of $K(n, X)$ by computors, that we have used this kind of argument in (VI).

\section{\$4. Certain properties of $K(n, X)$.}

On $H(X)$ we have by $(3.1)$ and (3.2) in (VI)

$$
\begin{aligned}
& H^{\prime}(X)=-\frac{1}{n-1}\left(\frac{n-X}{n-1}\right)^{n-2}\left\{\left(n^{2}-1\right) X^{2}-3 n X+n(n+2)\right\}+2 n+1, \\
& H^{\prime \prime}(X)=\frac{n}{n-1}\left(\frac{n-X}{n-1}\right)^{n-3}(X-1)\{(n+1) X-(n+4)\}
\end{aligned}
$$

and

$$
H(1)=H^{\prime}(1)=H^{\prime \prime}(1)=0 \text {. }
$$

Hence $H^{\prime}(X)$ is negative and decreasing in $1<X<(n+4) /(n+1)$ and increasing in $(n+4) /(n+1)<X<n$ and $H^{\prime}(n)=2 n+1>0$. Therefore, we can define a constant $a_{n}$ by 


$$
H^{\prime}\left(a_{n}\right)=0, \quad 1<a_{n}<n
$$

for each $n>2$. On $a_{n}$ we know the following fact ([18]).

Proposition 2. $H(X)$ is decreasing in $1<X<a_{n}$ and increasing in $a_{n}<X<n$ and $a_{n}$ has the properties:

$$
\begin{aligned}
& \frac{n+4}{n+1}<a_{n}<n \quad \text { for } n>2, \\
& a_{n}<\frac{2 n+10}{2 n+1} \quad \text { for } n>2.5, \\
& \frac{2 n+9}{2 n+1}<a_{n} \quad \text { for } n \geqq \frac{1+\sqrt{137}}{4}=3.17617 \cdots
\end{aligned}
$$

and $a_{n}$ is decreasing as function of $n$ in $3.5 \leqq n<\infty$.

LEMMA 4.1. $w(n, X)$ is increasing with respect to $n$ for $1<n(1<X<n)$ and decreasing with respect to $X$ for $1<X<n$.

Proof. Since we have

and

$$
\frac{\partial}{\partial n} \log \left(\frac{n-X}{n-1}\right)^{n-1}=\log \frac{n-X}{n-1}+\frac{X-1}{n-X} \rightarrow 0 \quad(\text { as } n \rightarrow \infty)
$$

$$
\frac{\partial^{2}}{\partial n^{2}} \log \left(\frac{n-X}{n-1}\right)^{n-1}=-\frac{(X-1)^{2}}{(n-1)(n-X)^{2}}<0 \quad \text { for } \quad 1<X<n,
$$

we obtain immediately this lemma.

Q.E.D.

LEMMA 4.2. $\quad P_{2}(x)$ and $P_{3}(x)$ are increasing with respect to $n$ for $1<n$ as functions of $n$ for each fixed $x(0 \leqq x \leqq 1)$.

Proof. From (1.6) we obtain

$$
\frac{\partial P_{2}(x)}{\partial n}=2 x^{2}-2(4 n+5) x+16(2 n-1),
$$

which becomes $24(n-1)(>0)$ at $x=1$ and $(4 n+5) / 2>1$. Hence we have $\partial P_{2}(x) / \partial n>0$ for $n>1$ and $0 \leqq x \leqq 1$.

Next, from (1.7) we obtain

$$
\frac{\partial P_{3}(x)}{\partial n}=-x^{3}+(4 n-7) x^{2}+(8 n-13) x+3(4 n-1)
$$

and

$$
\frac{\partial^{2} P_{3}(x)}{\partial x \partial n}=-3 x^{2}+2(4 n-7) x+8 n-13
$$

hence we have

$$
\left[\frac{\partial^{2} P_{3}(x)}{\partial x \partial n}\right]_{x=0}=8 n-13, \quad\left[\frac{\partial^{2} P_{3}(x)}{\partial x \partial n}\right]_{x=1}=16 n-30
$$

and 


$$
\left[\frac{\partial P_{3}(x)}{\partial n}\right]_{x=0}=3(4 n-1)>0, \quad\left[\frac{\partial P_{3}(x)}{\partial n}\right]_{x=1}=24(n-1)>0 .
$$

From these we see that if $n \geqq 13 / 8$, then

$$
\frac{\partial P_{3}(x)}{\partial n}>0 \quad \text { for } \quad 0 \leqq x \leqq 1 .
$$

If $1<n<13 / 8$, then $(4 n-7) / 3<-(1 / 6)$ and so $\partial^{2} P_{3}(x) / \partial x \partial n<0$ for $0 \leqq x \leqq 1$. Therefore, it holds the same inequality above.

Q.E.D.

LEMMA 4.3. $y(n, X)$ is increasing with respect to $n$ for $1<n(1<X<n)$ and decreasing with respect to $X$ for $1<X<n$.

Proof. We have

$$
\begin{aligned}
& \frac{\partial}{\partial n} \log \left(\frac{n-X}{n-1}\right)^{(n-1)(4 n+1) /(5 n-1)}=\frac{4\left(5 n^{2}-2 n+2\right)}{(5 n-1)^{2}} \log \frac{n-X}{n-1}+\frac{(4 n+1)(X-1)}{(5 n-1)(n-X)} \\
& \quad=\frac{4\left(5 n^{2}-2 n+2\right)}{(5 n-1)^{2}}\left[\log \frac{n-X}{n-1}+\frac{(X-1)(4 n+1)(5 n-1)}{4\left(5 n^{2}-2 n+2\right)(n-X)}\right] .
\end{aligned}
$$

We have

and

$$
\log \frac{n-X}{n-1}+\frac{(X-1)(4 n+1)(5 n-1)}{4\left(5 n^{2}-2 n+2\right)(n-X)} \rightarrow 0 \quad(\text { as } n \rightarrow \infty)
$$

$$
\begin{aligned}
-\frac{\partial}{\partial n} & {\left[\log \frac{n-X}{n-1}+\frac{(X-1)(4 n+1)(5 n-1)}{4\left(5 n^{2}-2 n+2\right)(n-X)}\right] } \\
& =\frac{X-1}{(n-1)(n-X)}-\frac{(X-1)\left\{100 n^{4}+10 n^{3}-57 n^{2}+4 n-2-45 n(n-2) X\right\}}{4\left(5 n^{2}-2 n+2\right)^{2}(n-X)^{2}}
\end{aligned}
$$

and

$$
\begin{aligned}
& 4\left(5 n^{2}-2 n+2\right)^{2}(n-X)-(n-1)\left(100 n^{4}+10 n^{3}-57 n^{2}+4 n-2\right)+45 n(n-1)(n-2) X \\
& =10 n^{4}+163 n^{3}-93 n^{2}+22 n-2-X\left(100 n^{4}-125 n^{3}+231 n^{2}-122 n+16\right) .
\end{aligned}
$$

Now, regarding the last expression we see that

$$
100 n^{4}-125 n^{3}+231 n^{2}-122 n+16>206 n^{2}-122 n+16>0 \text { for } n>1
$$

and so it is decreasing with respect to $X$ when $n>1$ and it becomes at $X=1$

$$
-90 n^{4}+288 n^{3}-324 n^{2}+144 n-18=-18(n-1)^{2}(5 n-1)<0 \text {. }
$$

Therefore it is negative for $1<X<n$, when $n>1$, from which we obtain

$$
\frac{\partial}{\partial n}\left[\log \frac{n-X}{n-1}+\frac{(X-1)(4 n+1)(5 n-1)}{4\left(5 n^{2}-2 n+2\right)(n-X)}\right]<0 \quad \text { for } \quad 1<X<n
$$

and so

$$
\log \frac{n-X}{n-1}+\frac{(X-1)(4 n+1)(5 n-1)}{4\left(5 n^{2}-2 n+2\right)(n-X)}>0
$$


and hence

$$
\frac{\partial}{\partial n} y(n, X)>0 \quad \text { for } \quad 1<X<n
$$

when $n>1$.

The second claim is evident.

Q.E.D.

LEMMA 4.4. $\lambda(y(n, X))-\tilde{\lambda}(X)$ is increasing with respect to $X(1<X<n)$, when $n>1$, and $\lambda(x)-\tilde{\lambda}(Y)$ is decreasing with respect to $n$ for $n>1$ with $0<x<1$ and $1<Y<n$.

Proof. The first part holds from Lemma 4.3 and the facts that $\lambda(x)$ is decreasing with respect to $x$ in $0<x<1$ and $\tilde{\lambda}(X)$ is decreasing with respect to $X$ in $1<X<n$.

Next, for fixed $x(0<x<1)$ and $Y(1<Y<n)$ we obtain from (1.8) and (1.9)

and

$$
\frac{\partial}{\partial n} \lambda(x)-\frac{\partial}{\partial n} \tilde{\lambda}(Y)=\frac{n+1-2 x}{(n-x)^{2}}-\frac{Y^{2}+n(n-3) Y+n}{(n-1)^{2} Y(n-Y)}
$$

Hence

$$
\begin{aligned}
& \frac{\partial}{\partial x} \frac{n+1-2 x}{(n-x)^{2}}=\frac{2(1-x)}{(n-x)^{3}}>0, \\
& \frac{\partial}{\partial Y} \frac{Y^{2}+n(n-3) Y+n}{Y(n-Y)}=\frac{n(Y-1)\{(n-2) Y+n\}}{Y^{2}(n-Y)^{2}}>0 .
\end{aligned}
$$

$$
\frac{n+1-2 x}{(n-x)^{2}}-\frac{Y^{2}+n(n-3) Y+n}{(n-1)^{2} Y(n-Y)}<\frac{n-1}{(n-1)^{2}}-\frac{1+n(n-3)+n}{(n-1)^{3}}=0,
$$

which implies the second claim.

Q.E.D.

LEMMA 4.5. $n(1-w)^{9}(1-X w) /(n-w)$ is increasing with respect to $X$ in $1<X<n$ with $n>1+1 / \sqrt{ } 2$ and decreasing with respect to $n$ in $1<n$ with $1<X<n$.

Proof. First we show $1-X w>0$. In fact from (3.9) we get

$$
\frac{\partial}{\partial X} X w=\left(\frac{n-X}{n-1}\right)^{n-1}-X\left(\frac{n-X}{n-1}\right)^{n-2}=-\frac{n(X-1)}{n-1}\left(\begin{array}{c}
n-X \\
n-1
\end{array}\right)^{n-2}<0
$$

and so

$$
1-X w>1-\lim _{X \rightarrow 1} X w=1-1=0 \text {. }
$$

Now, we have

$$
\begin{aligned}
\frac{\partial}{\partial X}-\frac{(1-w)^{3}(1-X w)}{n-w} & =-\frac{(1-w)^{2}\left\{3 n-1-2 w+X\left(n-4 n w+3 w^{2}\right)\right\}}{\partial w}-\frac{w(1-w)^{3}}{n-w} \\
& =\frac{(n-1) w(1-w)^{2}\left\{3 n-1-2 w+X\left(n-4 n w+3 w^{2}\right)\right\}}{(n-X)(n-w)^{2}}-\frac{w(1-w)^{3}}{n-w} \\
& =\frac{w(1-w)^{2}}{(n-X)(n-w)^{2}} \cdot \tilde{\rho}(w),
\end{aligned}
$$

where 


$$
\begin{aligned}
\tilde{\rho}(w):= & 2 n^{2}-4 n+1+\left(n^{2}-n+2\right) w-n w^{2} \\
& +X\left\{n^{2}-\left(4 n^{2}-3 n+1\right) w+(3 n-2) w^{2}\right\} .
\end{aligned}
$$

We shall show $\tilde{\rho}(w) \geqq 0$ for $0<w<1$, considering $X$ as function of $w$. Since we have

$$
\lim _{w \rightarrow 0} X(w)=n \text { and } \lim _{w \rightarrow 1} X(w)=1
$$

we obtain

$$
\tilde{\rho}(0)=2 n^{2}-4 n+1+n^{3}=(n-1)\left(n^{2}+3 n-1\right)>0, \quad \tilde{\rho}(1)=0 .
$$

Since we have

$$
\frac{\partial X}{\partial w}=-\frac{n-X}{(n-1) w}, \quad \frac{\partial^{2} X}{\partial w^{2}}=\frac{n-X}{(n-1) w^{2}}+\frac{1}{(n-1) w} \frac{\partial X}{\partial w},
$$

we obtain

$$
\left.\frac{\partial X}{\partial w}\right|_{w=1}=-1 \text { and }\left.\quad \frac{\partial^{2} X}{\partial w^{2}}\right|_{w=1}=\frac{n-2}{n-1} .
$$

We obtain from (4.7)

$$
\begin{aligned}
\frac{\partial \tilde{\rho}}{\partial w}= & n^{2}-n+2-2 n w+X\left\{-\left(4 n^{2}-3 n+1\right)+2(3 n-2) w\right\} \\
& -\frac{n-X}{(n-1) w}\left\{n^{2}-\left(4 n^{2}-3 n+1\right) w+(3 n-2) w^{2}\right\},
\end{aligned}
$$

from which we get

$$
\left.\frac{\partial \tilde{\rho}}{\partial w}\right|_{w=1}=0,\left.\quad \frac{\partial^{2} \tilde{\rho}}{\partial w^{2}}\right|_{w=1}=5 n(n-1)>0 .
$$

Hence, $\tilde{\rho}(w)$ is positive near $w=1$ in $0<w<1$.

Here, we suppose that $\tilde{\rho}(w) \geqq 0$ does not hold in $0<w<1$. Then there exist $\xi, \eta(0<\xi<\eta<1)$ such that

$$
\tilde{\rho}(\xi)=\tilde{\rho}(\eta)=0, \quad \tilde{\rho}^{\prime}(\xi) \leqq 0, \quad \tilde{\rho}^{\prime}(\eta) \geqq 0 .
$$

Since we have from (4.8)

$$
\begin{aligned}
(n-1) w \frac{\partial \tilde{\rho}}{\partial w}= & -n^{3}+\left(5 n^{3}-5 n^{2}+4 n-2\right) w-n(5 n-4) w^{2} \\
& +X\left\{n^{2}-\left(4 n^{3}-3 n^{2}+n\right) w+\left(6 n^{2}-7 n+2\right) w^{2}\right\} .
\end{aligned}
$$

On the other hand, regarding (4.7) we see that for $0<w<1$

$$
2 n^{2}-4 n+1+\left(n^{2}-n+2\right) w-n w^{2}>\min \left\{2 n^{2}-4 n+1,3(n-1)^{2}\right\},
$$

and so if $n \geqq 1+1 / \sqrt{2}=1.7071 \cdots$, then

$$
2 n^{2}-4 n+1+\left(n^{2}-n+2\right) w-n w^{2}>0 \quad \text { for } \quad 0<w<1 \text {, }
$$


and

$$
n^{2}-\left(4 n^{2}-3 n+1\right) w+(3 n-2) w^{2}<0 \quad \text { at } \quad w=\xi \text { and } \eta \text {. }
$$

Eliminating $X$ from (4.7) and $\left(4.8^{\prime}\right)$ at $w=\xi, \eta$, we obtain respectively

$$
\begin{aligned}
& \left\{n^{2}-\left(4 n^{2}-3 n+1\right) w+(3 n-2) w^{2}\right\}\left\{-n^{3}+\left(5 n^{3}-5 n^{2}+4 n-2\right) w-n(5 n-4) w^{2}\right\} \\
& -\left\{2 n^{2}-4 n+1+\left(n^{2}-n+2\right) w-n w^{2}\right\}\left\{n^{2}-\left(4 n^{3}-3 n^{2}+n\right) w+\left(6 n^{2}-7 n+2\right) w^{2}\right\} \\
& \geqq 0 \quad \text { at } w=\xi \text { and } \leqq 0 \text { at } w=\eta,
\end{aligned}
$$

when $n \geqq 1+1 / \sqrt{2}$. The left hand side of the above inequalities can be expressed as

$$
\begin{aligned}
n(n-1)[ & -n\left(n^{2}+3 n-1\right)+\left(17 n^{3}-14 n^{2}+10 n-1\right) w-\left(16 n^{3}+8 n^{2}-13 n+7\right) w^{2} \\
& \left.+\left(25 n^{2}-15 n+2\right) w^{3}-(9 n-6) w^{4}\right] .
\end{aligned}
$$

Here we set for simplicity

$$
\begin{aligned}
R_{4}(x):= & 3(3 n-2) x^{4}-\left(25 n^{2}-15 n+2\right) x^{3}+\left(16 n^{3}+8 n^{2}-13 n+7\right) x^{2} \\
& -\left(17 n^{3}-14 n^{2}+10 n-1\right) x+n\left(n^{2}+3 n-1\right),
\end{aligned}
$$

then it must hold

$$
R_{4}(\xi) \leqq 0 \quad \text { and } \quad R_{4}(\eta) \geqq 0
$$

We have

$$
\begin{aligned}
& R_{4}{ }^{\prime}(x)= 12(3 n-2) x^{3}-3\left(25 n^{2}-15 n+2\right) x^{2}+2\left(16 n^{3}+8 n^{2}-13 n+7\right) x \\
&-17 n^{3}+14 n^{2}-10 n+1 \\
& \frac{1}{2} R_{4}^{\prime \prime}(x)=18(3 n-2) x^{2}-3\left(25 n^{2}-15 n+2\right) x+16 n^{3}+8 n^{2}-13 n+7 .
\end{aligned}
$$

Since we have

and

$$
R_{4}^{\prime \prime}(0)=16 n^{3}+8 n^{2}-13 n+7>0, \quad R_{4}^{\prime \prime}(1)=2(n-1)^{2}(16 n-35)
$$

$$
R_{4}^{\prime}(0)=-\left(17 n^{3}-14 n^{2}+10 n-1\right)<0, \quad R_{4}^{\prime}(1)=15(n-1)^{3}>0,
$$

therefore $R_{4}(x)$ must be decreasing first and then turns into increasing in $0<x<1$. We have

$$
R_{4}(0)=n\left(n^{2}+3 n-1\right)>0 \quad \text { and } \quad R_{4}(1)=0 .
$$

From these facts it is impossible to have (4.10), when $n \geqq 1+1 / \sqrt{2}$. Thus we obtain

$$
\tilde{\rho}(w) \geqq 0 \quad \text { for } \quad 0<w<1 \text {, when } n \geqq 1+1 / \sqrt{2} \text {. }
$$

Finally, regarding the second part we have 


$$
\begin{aligned}
\frac{\partial}{\partial n} \log \frac{n(1-w)^{3}(1-X w)}{n-w} & =\frac{1}{n}-\frac{1}{n-w}+\left(-\frac{3}{1-w}-\frac{X}{1-X w}+\frac{1}{n-w}\right) \frac{\partial w}{\partial n} \\
& =-\frac{w}{n(n-w)}-\left\{\frac{3 n-1-2 w}{(1-w)(n-w)}+\frac{1}{1-X w}\right\} \frac{\partial w}{\partial n}<0
\end{aligned}
$$

by Lemma 4.1 , when $n>1$.

LEMMA 4.6. $f_{0}(x) / B=2 n-1-x-(n-x /(n-1))^{n-1}\left\{n-x+(n-1) x^{2}\right\}$ is increasing with respect to $x$ in $0<x<1$ with $n>1$ and decreasing with respect to $n$ in $2<n$ with $0<x<1$.

Proof. The first part is evident from Lemma 3.4. We have

$$
\begin{array}{r}
\frac{\partial}{\partial n} \frac{f_{0}(x)}{B}=2-\left(\frac{n-x}{n-1}\right)^{n-1}\left[\left(1+x^{2}\right)+(\right. \\
\left(\log \frac{n-x}{n-1}-\frac{1-x}{n-x}\right) \\
\left.\times\left\{n-x+(n-1) x^{2}\right\}\right]
\end{array}
$$

and

$$
\begin{aligned}
& \frac{(n-1)^{n-1}}{(n-x)^{n-3}} \frac{\partial^{2}}{\partial x \partial n} \frac{f_{0}(x)}{B}=n(1-x)^{2}(n-2 x) \\
& \quad+(n-x)\left\{n^{2}-n(2 n-1) x+\left(n^{2}-1\right) x^{2}\right\}\left(\log \frac{n-x}{n-1}-\frac{1-x}{n-x}\right) .
\end{aligned}
$$

We see easily the following inequalities

$$
\begin{array}{r}
n(1-x)^{2}(n-2 x)>0 \text { and } n^{2}-n(2 n-1) x+\left(n^{2}-1\right) x^{2}>0 \\
\text { for } 0<x<1 \text { and } n>2 .
\end{array}
$$

On the other hand we have

$$
\lim _{n \rightarrow \infty}\left(\log \frac{n-x}{n-1}-\frac{1-x}{n-x}\right)=0
$$

and

$$
\frac{\partial}{\partial n}\left(\log \frac{n-x}{n-1}-\frac{1-x}{n-x}\right)=-\frac{(1-x)^{2}}{(n-1)(n-x)^{2}}<0
$$

hence

$$
\log \frac{n-x}{n-1}-\frac{1-x}{n-x}>0
$$

Thus we see that

$$
\frac{\partial^{2}}{\partial x \partial n} \frac{f_{0}(x)}{B}>0 \quad \text { for } 0<x<1 \text { and } n>2
$$

and so

$$
\frac{\partial}{\partial n} \frac{f_{0}(x)}{B}<\lim _{x \rightarrow 1} \frac{\partial}{\partial n} \frac{f_{0}(x)}{B}=0 . \quad \text { Q.E.D. }
$$

LEMMA $4.7 Q_{2}(X)$ is decreasing with respect to $X$ in $1 \leqq X \leqq 2.5$ with $n \geqq 3$ 
and increasing with respect to $n$ in $3 \leqq n$ with $1 \leqq X \leqq 2.5$, and positwe there.

Proof. From (3.17) we get for $1 \leqq X \leqq 2.5$

and

$$
\begin{aligned}
Q_{2}{ }^{\prime}(X) & =2\left(4 n^{2}+2 n-3\right) X-\left(4 n^{3}+18 n^{2}-19 n+3\right) \\
& \leqq 5\left(4 n^{2}+2 n-3\right)-\left(4 n^{3}+18 n^{2}-19 n+3\right) \\
& =-\left(4 n^{3}-2 n^{2}-29 n+18\right)<0 \quad \text { for } n \geqq 3
\end{aligned}
$$

$$
Q_{2}(2.5)=6 n^{3}-36 n^{2}+63 n-26.25 \geqq 9 n-26.25>0 \text { for } n \geqq 3 \text {. }
$$

From these we obtain the first part and

$$
Q_{2}(X)>0 \quad \text { for } \quad 1 \leqq X \leqq 2.5 \text {, when } n \geqq 3 \text {. }
$$

Next, we have

$$
\frac{\partial Q_{2}(X)}{\partial n}=2(4 n+1) X^{2}-\left(12 n^{2}+36 n-19\right) X+48 n^{2}-32 n+3
$$

and

$$
\frac{12 n^{2}+36 n-19}{4(4 n+1)}>3 \quad \text { for } n \geqq 3,
$$

and then $\partial Q_{2}(X) / \partial n$ is decreasing with respect to $X$ in $1<X<3$. Since we have

$$
\left.\frac{\partial Q_{2}(X)}{\partial n}\right|_{X=2.5}=18 n^{2}-72 n+63 \geqq 9 \quad \text { for } n \geqq 3 \text {, }
$$

we obtain

$$
\frac{\partial Q_{2}(X)}{\partial n}>0 \quad \text { for } n \geqq 3 \text { with } 1 \leqq X \leqq 2.5 . \quad \text { Q.E. D. }
$$

LEMMA 4.8. (i) $Q_{3}(X)<0$ for $1 \leqq X \leqq 2.5$, with $n \geqq 3.5$.

(ii) $Q_{3}(X)$ is decreasing with respect to $X$ in $1<X<2.2$, with $n \geqq 4.46$, and is decreasing in $1<X<\gamma_{n}$ and increasing in $\gamma_{n}<X<2.5$, with $3.5 \leqq n<4.46$, where

$$
\gamma_{n}:=\frac{2 n^{3}-9 n^{2}+13 n-3+\sqrt{4 n^{6}-12 n^{5}-23 n^{4}+66 n^{3}-11 n^{2}-24 n+9}}{3(n-1)(2 n-3)}
$$

(iii) $Q_{3}(X)$ is decreasing with respect to $n$ in $3 \leqq n$, with $1 \leqq X \leqq 2.5$.

Proof. Proof of (i). We get from (3.18)

$$
\begin{aligned}
Q_{3}(X)= & \left(2 n^{2}-5 n+3\right) X^{3}-\left(2 n^{3}-9 n^{2}+13 n-3\right) X^{2}-2 n\left(2 n^{2}-8 n+3\right) X-3 n^{2}(2 n-1) \\
= & -\left(2 X^{2}+4 X+6\right) n^{3}+\left(2 X^{3}+9 X^{2}+16 X+3\right) n^{2}-\left(5 X^{3}+13 X^{2}+6 X\right) n \\
& +3 X^{3}+3 X^{2}, \\
Q_{3}{ }^{\prime}(X)= & 3\left(2 n^{2}-5 n+3\right) X^{2}-2\left(2 n^{3}-9 n^{2}+13 n-3\right) X-2 n\left(2 n^{2}-8 n+3\right), \\
Q_{3}(1)= & -6(n-1)^{2}(2 n-1)<0,
\end{aligned}
$$


396

TOMINOSUKE OTSUKI

$$
\begin{aligned}
& Q_{3}{ }^{\prime}(1)=-\left(8 n^{3}-40 n^{2}+47 n-15\right)<0 \quad \text { for } n \geqq 3.5, \\
& Q_{3}(2.5)=-28.5 n^{3}+130.5 n^{2}-174.375 n+65.625<0 \quad \text { for } n \geqq 3,
\end{aligned}
$$

which imply $Q_{3}(X)<0$ for $1 \leqq X \leqq 2.5$, with $n \geqq 3.5$.

Proof of (ii). We have

$$
\begin{aligned}
Q_{3}{ }^{\prime}(2.2) & =-\left(12.8 n^{3}-84.64 n^{2}+135.8 n-56.76\right) \\
& \leqq-12.91808 n+56.76 \leqq-0.8546368 \text { for } n \geqq 4.46,
\end{aligned}
$$

which implies $Q_{3}{ }^{\prime}(X)<0$ in $1 \leqq X \leqq 2.2$ with the above evaluation of $Q_{\mathbf{3}}{ }^{\prime}(1)$. (Here we notice $\gamma_{4.4}=2.1803 \cdots<2.2$ ).

Now, for $n \geqq 3.5$, let $\gamma_{n}$ be the large root of the quadratic equation $Q_{3}{ }^{\prime}(X)$ $=0$ of $X$, then we obtain its expression above. The rest part is evident. (Here, we notice

$$
\left.\gamma_{3.5}=\frac{18+\sqrt{219}}{30}=1.093 \cdots, \quad \gamma_{4}=\frac{33+\sqrt{2196}}{45}=1.7682 \cdots\right) .
$$

Proof of (iii). We have from (3.18)

$$
\begin{aligned}
& \frac{\partial Q_{3}(X)}{\partial n}=-3\left(2 X^{2}+4 X+6\right) n^{2}+2\left(2 X^{3}+9 X^{2}+16 X+3\right) n-\left(5 X^{3}+13 X^{2}+6 X\right), \\
& \frac{\partial^{2} Q_{3}(X)}{\partial X \partial n}=-12(X+1) n^{2}+2\left(6 X^{2}+18 X+16\right) n-\left(15 X^{2}+26 X+6\right), \\
& \left.\frac{\partial^{2} Q_{3}(X)}{\partial X \partial n}\right|_{X=1}=-\left(24 n^{2}-80 n+47\right)<0, \\
& \left.\frac{\partial Q_{3}(X)}{\partial n}\right|_{X=1}=-12(n-1)(3 n-2)<0, \\
& \left.\frac{\partial Q_{3}(X)}{\partial n}\right|_{X=2.5}=-85.5 n^{2}+261 n-174.375<0 \quad \text { for } n \geqq 3,
\end{aligned}
$$

which imply easily

$$
\frac{\partial Q_{3}(X)}{\partial n}<0 \quad \text { for } 1 \leqq X \leqq 2.5 \text { and } n \geqq 3 \text { Q.E.D. }
$$

LEMMA 4.9. $X /(X-1)^{3} \sqrt{n-X}$ is decreasing with respect to $X$ in $1<X \leqq 2.5$ with $n \geq 3$.

Proof. We have easily

$$
\frac{\partial}{\partial X} \frac{X}{(X-1)^{3} \sqrt{n-X}}=-\frac{-5 X^{2}+(4 n-1) X+2 n}{2(X-1)^{4}(n-X)^{3 / 2}}
$$

and

$$
\begin{aligned}
& {\left[-5 X^{2}+(4 n-1) X+2 n\right]_{X=1}=6(n-1)>0,} \\
& {\left[-5 X^{2}+(4 n-1) X+2 n\right]_{X=2.5}=12 n-33.75 \geqq 2.25 \text { for } n \geqq 3 .}
\end{aligned}
$$


Hence we obtain

$$
-5 X^{2}+(4 n-1) X+2 n>0 \quad \text { in } \quad 1 \leqq X \leqq 2.5 \text { with } n \geqq 3 .
$$

Q.E.D.

Proposition 3. $K(n, X)$ defined by (3.16) satisfies the following inequality: Let $n \geqq 3$ and $1<X<b_{n}$, and $n_{1}, n_{2}, X_{1}, X_{2}$ be such that

then

$$
3 \leqq n_{1} \leqq n \leqq n_{2}, \quad a_{n} \leqq X_{1} \leqq X \leqq X_{2}<b_{n},
$$

where

$$
K(n, X) \geqq K\left(n_{1}, n_{2} ; X_{1}, X_{2}\right),
$$

$$
\begin{gathered}
K\left(n_{1}, n_{2} ; X_{1}, X_{2}\right)=\frac{\left(w\left(n_{1}, X_{2}\right)\right)^{2} \sqrt{n_{1}-w\left(n_{1}, X_{2}\right)}}{\left(1-w\left(n_{1}, X_{2}\right)\right)^{5}} \\
\times\left[\left\{-P_{2}\left(n_{2}, y\left(n_{2}, X_{1}\right)\right)+\left(\frac{n_{1}-y\left(n_{2}, X_{1}\right)}{n_{1}-1}\right)^{n_{1}-1} P_{3}\left(n_{1}, y\left(n_{2}, X_{1}\right)\right)\right\}\right. \\
\quad \times\left\{\log \frac{n_{2}-y\left(n_{2}, X_{1}\right)}{n_{2}-X_{1}}+\frac{n_{2} X_{1} y\left(n_{2}, X_{1}\right)-\left(2 n_{2}-1\right) X_{1}+n_{2}-y\left(n_{2}, X_{1}\right)}{\left(n_{2}-1\right) X_{1}\left(n_{2}-y\left(n_{2}, X_{1}\right)\right)}\right. \\
\left.\quad+\frac{2 n_{2}\left\{1-w\left(n_{2}, X_{1}\right)\right\}^{3}\left\{1-X_{1} w\left(n_{2}, X_{1}\right)\right\}}{n_{2}-w\left(n_{2}, X_{1}\right)}\right] \\
+\frac{3\left(y\left(n_{2}, X_{1}\right)\right)^{2}}{\left(1-y\left(n_{2}, X_{1}\right)\right)^{3} \sqrt{n_{1}-y\left(n_{2}, X_{1}\right)}\left[2 n_{2}-1-w\left(n_{1}, X_{2}\right)-\left(\frac{n_{2}-w\left(n_{1}, X_{2}\right)}{n_{2}-1}\right)^{n_{2}-1}\right.} \\
\left.\quad \times\left\{n_{2}-w\left(n_{1}, X_{2}\right)+\left(n_{2}-1\right)\left(w\left(n_{1}, X_{2}\right)\right)^{2}\right\}\right] \\
-\frac{X_{1} Q_{2}\left(n_{2}, X_{1}\right)}{\left(n_{1}-1\right)\left(X_{1}-1\right)^{3} \sqrt{n_{1}-X_{1}}}-\frac{X_{2} w\left(n_{1}, X_{2}\right) Q_{3}\left(n_{1}, X^{*}\right)}{\left(n_{2}-1\right)\left(X_{2}-1\right)^{3} \sqrt{n_{2}-X_{2}}}
\end{gathered}
$$

and $X^{*}=X_{1}$ for $n \geqq 4.46$ and $X^{*}=X_{2}$ when $X>\gamma_{n}$ and $X^{*}=X_{1}$ when $X \leqq \gamma_{n}$ for $(8+\sqrt{34}) / 4 \leqq n<4.46$, and $X^{*}=X_{2}$ for $3 \leqq n<(8+\sqrt{34}) / 4$ and $P_{2}(n, x), P_{3}(n, x)$, $Q_{2}(n, x), Q_{3}(n, x)$ stand for $P_{2}(x), P_{3}(x), Q_{2}(x), Q_{3}(x)$ with $n$ by (1.6), (1.7), (3.17), (3.18) respectively.

Proof. By Lemma 4.1 and Lemma 4.2 we have first

$$
\begin{aligned}
& w\left(n_{1}, X_{2}\right) \leqq w(n, X) \leqq w\left(n_{2}, X_{1}\right), \\
& y\left(n_{1}, X_{2}\right) \leqq y(n, X) \leqq y\left(n_{2}, X_{1}\right) .
\end{aligned}
$$

From Lemma 3.2 we obtain

$$
\frac{(w(n, X))^{2} \sqrt{n-w(n, X)}}{(1-w(n, X))^{5}} \geqq \frac{\left(w\left(n_{1}, X_{2}\right)\right)^{2} \sqrt{n_{1}-w\left(n_{1}, X_{2}\right)}}{\left(1-w\left(n_{1}, X_{2}\right)\right)^{5}}>0 .
$$

From Lemma 3.1 and the above inequalities (4.13) we have

$$
-P_{2}(n, y(n, X))+\left(\frac{n-y(n, X)}{n-1}\right)^{n-1} P_{3}(n, y(n, X))
$$




$$
\geqq-P_{2}\left(n, y\left(n_{2}, X_{1}\right)\right)+\left(\frac{n-y\left(n_{2}, X_{1}\right)}{n-1}\right)^{n-1} P_{3}\left(n, y\left(n_{2}, X_{1}\right)\right)
$$

(by Lemma 4.2 )

$$
\geqq-P_{2}\left(n_{2}, y\left(n_{2}, X_{1}\right)\right)+\left(\frac{n-y\left(n_{2}, X_{1}\right)}{n-1}\right)^{n-1} P_{3}\left(n_{1}, y\left(n_{2}, X_{1}\right)\right) .
$$

On the other hand we see easily that $w(n, x)=(n-x /(n-1))^{n-1}$ with $0<x<1$ is increasing with respect to $n(>1)$ by the same argument in Lemma 4.1. Hence we obtain

$$
\begin{aligned}
& -P_{2}(n, y(n, X))+\left(\frac{n-y(n, X)}{n-1}\right)^{n-1} P_{3}(n, y(n, X)) \\
\geqq & -P_{2}\left(n_{2}, y\left(n_{2}, X_{1}\right)\right)+\left(\frac{n_{1}-y\left(n_{2}, X_{1}\right)}{n_{1}-1}\right)^{n_{1}-1} P_{3}\left(n_{1}, y\left(n_{2}, X_{1}\right)\right) .
\end{aligned}
$$

Next, denoting $\lambda(x)$ and $\tilde{\lambda}(x)$ by (1.8) and (1.9) by $\lambda_{n}(x)$ and $\tilde{\lambda}_{n}(x)$ respectively, by Lemma 4.4 we obtain

$$
\begin{aligned}
& \lambda_{n}(y(n, X))-\tilde{\lambda}_{n}(X) \geqq \lambda_{n}\left(y\left(n_{2}, X_{1}\right)-\tilde{\lambda}_{n}\left(X_{1}\right)\right. \\
& \geqq \lambda_{n_{2}}\left(y\left(n_{2}, X_{1}\right)-\tilde{\lambda}_{n_{2}}\left(X_{1}\right)\right. \\
& =\log \frac{n_{2}-y\left(n_{2}, X_{1}\right)}{n_{2}-X_{1}}+\frac{n_{2} X_{1} y\left(n_{2}, X_{1}\right)-\left(2 n_{2}-1\right) X_{1}+n_{2}-y\left(n_{2}, X_{1}\right)}{\left(n_{2}-1\right) X_{1}\left(n_{2}-y\left(n_{2}, X_{1}\right)\right)}>0 .
\end{aligned}
$$

From Lemma 4.5 we obtain

$$
\frac{n(1-w(n, X))^{3}(1-X w(n, X))}{n-w(n, X)} \geqq \frac{n_{2}\left(1-w\left(n_{2}, X_{1}\right)\right)^{3}\left(1-X_{1} w\left(n_{2}, X_{1}\right)\right)}{n_{2}-w\left(n_{2}, X_{1}\right)}>0 .
$$

Then, we proceed to the second part of (3.16). Noticing $f_{0}(x)<0$ for $0<x<1$, by (4.12), (4.13) and Lemma 4.6 we obtain

$$
\begin{aligned}
\frac{(y(n, X))^{2}}{(1-y(n, X))^{3} \sqrt{n-y(n, X)}} \cdot & {\left[2 n-1-w(n, X)-\left(\frac{n-w(n, X)}{n-1}\right)^{n-1}\right.} \\
& \left.\times\left\{n-w(n, X)+(n-1)(w(n, X))^{2}\right\}\right] \\
\geqq \frac{\left(y\left(n_{2}, X_{1}\right)^{2}\right.}{\left(1-y\left(n_{2}, X_{1}\right)^{2} \sqrt{n_{1}-y\left(n_{2},\right.}\right.} & =\left[2 n_{1}-w\left(n_{1}, X_{2}\right)-\left(\frac{n_{2}-w\left(n_{1}, X_{2}\right)}{n_{2}-1}\right)^{n_{2}-1}\right. \\
\times & \left.\left\{n_{2}-w\left(n_{1}, X_{2}\right)+\left(n_{2}-1\right)\left(w\left(n_{1}, X_{2}\right)\right)^{2}\right\}\right] .
\end{aligned}
$$

Lastly we deal with the third part of (3.16). By Lemma 4.7, Lemma 4.8 and Lemma 4.9 we obtain

$$
-\frac{X}{(n-1)(X-1)^{3} \sqrt{n-X}} \cdot\left[Q_{2}(n, X)+w(n, X) Q_{3}(n, X)\right]
$$




$$
\begin{aligned}
& \geqq-\frac{X_{1}}{\left(n_{1}-1\right)\left(X_{1}-1\right)^{3} \sqrt{ } n_{1}-X_{1}} \cdot Q_{2}\left(n_{2}, X_{1}\right) \\
& \quad-\frac{X_{2}}{\left(n_{2}-1\right)\left(X_{2}-1\right)^{3} \sqrt{ } n_{2}-X_{2}} \cdot w\left(n_{1}, X_{2}\right) Q_{3}\left(n_{1}, X^{*}\right),
\end{aligned}
$$

where $X^{*}=X_{1}$ for $n \geqq 4.46$, and $X^{*}=X_{1}$ when $X \leqq \gamma_{n}$ and $X^{*}=X_{2}$ when $X>\gamma_{n}$ for $(8+\sqrt{ } 34) / 4 \leqq n<4.46$, and $X^{*}=X_{2}$ for $3 \leqq n<(8+\sqrt{ } 34) / 4$.

Using these inequalities $(4.14) \sim(4.19)$, we obtain

$$
K(n, X) \geqq K\left(n_{1}, n_{2} ; X_{1}, X_{2}\right) .
$$

Q.E.D.

§5. Evaluation of $V\left(x, X_{n}(x)\right)$ near $x=1$.

Refering to the argument used in $\$ 8$ of (III), we shall try more fine evaluation of $V\left(x, X_{n}(x)\right)$ near $x=1$ than it.

LEMMA 5.1. When $4 \leqq n \leqq 10$, we have

$$
\begin{aligned}
U_{0}(x)> & \frac{n^{2}\left(n^{2}-n+1\right)(n-1)^{n-7 / 2}}{6} \cdot(X-1)\left\{1-\frac{(12 n-13) p_{n}+6 n-10}{6(n-1)}(X-1)\right. \\
& \left.+\frac{(4 n-5) p_{n}\left(p_{n}+3 n-5\right)}{6(n-1)^{2}}-(X-1)^{2}\right\} \quad \text { for } 1<X \leqq C \leqq \min \left(2, b_{n}\right),
\end{aligned}
$$

where if $C=\min \left(2, b_{n}\right)$, then $p_{n}=e_{n-2}=(1+1 /(n-2))^{n-2}$; if $4 \leqq n \leqq 4.5$ and $C=1.225$, then $p_{n}=((n-1) /(n-1.225))^{n-1.169}$; and if $4.5 \leqq n \leqq 5$ and $C=1.163$, then $p_{n}=$ $((n-1) /(n-1.2))^{n-1.165}, X=X_{n}(x)$.

Proof. We obtain $F_{2}(1)=F_{2}{ }^{\prime}(1)=F_{2}{ }^{\prime \prime}(1)=F_{2}{ }^{\prime \prime}(1)=0$ from $(3.1) \sim(3.5)$ and so for $x(0<x<1)$ there exists $x_{1}\left(x<x_{1}<1\right)$ such that

$$
F_{2}(x)=-\frac{n(n-1)}{24}(x-1)^{4} \tilde{R}_{3}\left(x_{1}\right)
$$

by (3.6). We have from (3.7)

$$
\begin{aligned}
\tilde{R}_{3}{ }^{\prime}(x)= & 12 n^{4}-19 n^{3}+48 n^{2}-35 n-18-2\left(2 n^{4}+3 n^{3}+11 n^{2}-6 n-16\right) x \\
& +3(n-1)(n+1)(n+2) x^{2}>0,
\end{aligned}
$$

$$
\tilde{R}_{3}^{\prime}(0)>0, \quad \tilde{R}_{3}^{\prime}(1)>0, \quad \frac{2 n^{4}+3 n^{3}+11 n^{2}-6 n-16}{3(n-1)(n+1)(n+2)}>1,
$$

when $n \geqq 2$. Therefore we have

$$
\tilde{R}_{3}(x)<\tilde{R}_{3}(1)=-4(n-1)^{2}\left(n^{2}-n+1\right)<0,
$$

and hence we obtain the inequality:

$$
F_{2}(x)>\frac{1}{6} n(n-1)^{n-2}\left(n^{2}-n+1\right)(1-x)^{4} \quad \text { for } \quad 0<x<1 .
$$


Now, since we have from (1.8) and (1.9)

$$
\begin{aligned}
& \lambda^{\prime}(x)=-\frac{1-x}{(n-x)^{2}}, \quad \tilde{\lambda}^{\prime}(x)=-\frac{(x-1)\{n+(n-1) x\}}{(n-1) x^{2}(n-x)}, \\
& \tilde{\lambda}^{\prime \prime}(x)=-\frac{2}{(n-1) x^{3}}-\frac{1}{(n-x)^{2}}, \quad \frac{d X}{d x}=-\frac{(1-x)(n-x)^{n-2}}{(X-1)(n-X)^{n-2}},
\end{aligned}
$$

we can obtain

$$
\frac{d}{d X}\{\lambda(x)-\tilde{\lambda}(X)\}=\frac{(X-1)(n-X)^{n-2}}{(n-x)^{n}}+\frac{(X-1)\{n+(n-1) X\}}{(n-1) X^{2}(n-X)},
$$

which becomes 0 at $X=1$, and

$$
\begin{aligned}
\frac{d^{2}}{d X^{2}}\{\lambda(x)-\tilde{\lambda}(X)\}= & \frac{(n-1)(2-X)(n-X)^{n-3}}{(n-x)^{n}}-\frac{n(X-1)^{2}(n-X)^{2 n-4}}{(1-x)(n-x)^{2 n-1}} \\
& +\frac{2}{(n-1) X^{3}}+\frac{1}{(n-X)^{2}},
\end{aligned}
$$

and

$$
\begin{gathered}
\frac{d}{d X} \frac{(2-X)(n-X)^{n-3}}{(n-x)^{n}}=-\frac{(n-2)(3-X)(n-X)^{n-4}}{(n-x)^{n}}-\frac{n(X-1)(2-X)(n-X)^{2 n-5}}{(1-x)(n-x)^{2 n-1}} \\
-\frac{(n-2) 2(n-1)^{n-4}}{(n-1)^{n}}-\frac{n(n-1)^{2 n-5}}{(n-1)^{2 n-1}} p_{n}=-\frac{2(n-2)+n p_{n}}{(n-1)^{4}}
\end{gathered}
$$

where $p_{n}$ denotes the right hand side of (i), (ii) and (iii) in Lemma 2.5 according to these cases. Hence we obtain

$$
\frac{(n-1)(2-X)(n-X)^{n-3}}{(n-x)^{n}}>\frac{1}{(n-1)^{2}}-\frac{2(n-2)+n p_{n}}{(n-1)^{3}}(X-1) \text {. }
$$

We obtain also

$$
\frac{n(X-1)^{2}(n-X)^{2 n-4}}{(1-x)(n-x)^{2 n-1}}<\frac{n(X-1)(n-1)^{2 n-4}}{(n-1)^{2 n-1}} p_{n}=\frac{n p_{n}}{(n-1)^{3}}(X-1)
$$

and

$$
\frac{2}{(n-1) X^{3}}>\frac{2}{n-1}-\frac{6}{n-1}(X-1), \quad \frac{1}{(n-X)^{2}}>\frac{1}{(n-1)^{2}}+\frac{2}{(n-1)^{3}}(X-1) .
$$

Using these inequalities in the right hand side of the above equality, we obtain for $n \geqq 4$

$$
\frac{d^{2}}{d X^{2}}\{\lambda(x)-\tilde{\lambda}(X)\}>\frac{2 n}{(n-1)^{2}}-\frac{2 n\left(p_{n}+3 n-5\right)}{(n-1)^{3}}(X-1) .
$$

which implies 


$$
\lambda(x)-\tilde{\lambda}(X)>\frac{n}{(n-1)^{2}}(X-1)^{2}-\frac{n\left(p_{n}+3 n-5\right)}{3(n-1)^{3}}(X-1)^{3}
$$

Last, since we have

$$
\text { for } 1<X \leqq C \text {. }
$$

$$
\frac{d}{d X} x^{2} \sqrt{n-x}=-\frac{x(4 n-5 x)(n-X)^{n-2}}{2(n-x)^{n-3 / 2}} \cdot \frac{X-1}{1-x},
$$

we obtain the following inequality:

$$
x^{2} \sqrt{n-x}>\sqrt{n-1}-\frac{(4 n-5) p_{n}}{2 \sqrt{n-1}}(X-1) \quad \text { for } \quad 1<X \leqq C .
$$

Using these inequalities $(5.1) \sim(5.3)$ for the expression of $U_{0}(x)$ in (1.16) and $X-1>1-x$ by Proposition 4 in (IV), we obtain

$$
\begin{aligned}
& U_{0}(x)=\frac{x^{2} \sqrt{n-x}}{(1-x)^{5}} F_{2}(x)\{\lambda(x)-\tilde{\lambda}(X(x))\} \\
& >\frac{n^{2}\left(n^{2}-n+1\right)(n-1)^{n-7 / 2}}{6}\left\{1-\frac{(4 n-5) p_{n}}{2(n-1)}(X-1)\right\}\left\{1-\frac{p_{n}+3 n-5}{3(n-1)}(X-1)\right\}(X-1),
\end{aligned}
$$

which becomes the required one in the statement of the lemma. Q.E.D.

We prepare some formulas on $f_{0}(x)$. We obtain from (1.3)

$$
\left\{\begin{array}{l}
f_{0}(x)=(2 n-1-x) B-(n-x)^{n-1}\left\{(n-1) x^{2}-x+n\right\}, \\
f_{0}{ }^{\prime}(x)=-B+(n-x)^{n-2}\left\{\left(n^{2}-1\right) x^{2}-n(2 n-1) x+n^{2}\right\} \\
f_{0}^{\prime \prime}(x)=-n(n-1)(x-1)\{(n+1) x-3 n\}(n-x)^{n-3}, \\
f_{0}^{\prime \prime \prime}(x)=n(n-1)\left\{\left(n^{2}-1\right) x^{2}-\left(6 n^{2}-5 n-2\right) x+n(7 n-8)\right\}(n-x)^{n-4}, \\
f_{0}^{(4)}(x)=-n(n-1)(n-2)\left\{\left(n^{2}-1\right) x^{2}-\left(8 n^{2}-7 n-3\right) x+n(13 n-15)\right\}(n-x)^{n-5}, \\
f_{0}^{(5)}(x)=n(n-1)(n-2)(n-3)\left\{\left(n^{2}-1\right) x^{2}-\left(10 n^{2}-9 n-4\right) x+3 n(7 n-8)\right\}(n-x)^{n-6}, \\
f_{0}{ }^{(6)}(x)=-n(n-1)(n-2)(n-3)(n-4)\left\{\left(n^{2}-1\right) x^{2}-\left(12 n^{2}-11 n-5\right) x+n(31 n-35)\right\} \\
\quad \times(n-x)^{n-7} .
\end{array}\right.
$$

LEMMA 5.2. When $3 \leqq n$, we have

$$
\begin{aligned}
U_{5}(x)> & \frac{n(2 n-1)(n-1)^{n-5 / 2}}{2}+\frac{n\left(5 n^{2}-3 n+1\right)(n-1)^{n-7 / 2}}{4}(X-1) \\
+ & \left.\frac{n(n-2)(n-3) q_{n}(n-1)^{1 / 2}\left(n-\varepsilon_{0}\right)^{n-6}}{40}-\frac{n(n-2)(3 n-1)(4 n-3)(n-1)^{n-9 / 2}}{8}\right\} \\
& \times(X-1)^{2}+\frac{n(n-2)(n-3)(4 n-3) q_{n}\left(n-\varepsilon_{0}\right)^{n-6}}{80 \sqrt{n-1}}(X-1)^{3} \\
& \text { for } 1<X \leqq C(\leqq 2),
\end{aligned}
$$


where we set

$$
q_{n}=q_{n}(C)=\left(n^{2}-1\right) C^{2}-\left(10 n^{2}-9 n-4\right) C+3 n(7 n-8)
$$

and $\varepsilon_{0}=1$ for $3 \leqq n<6, \varepsilon_{0}=2$ for $n \geqq 6$.

Proof. We have $f_{0}(1)=f_{0}^{\prime}(1)=f_{0}^{\prime \prime}(1)=0$ and

$$
f_{0}^{m}(1)=n(2 n-1)(n-1)^{n-2}, \quad f_{0}^{(3)}(1)=-2 n(n-2)(3 n-1)(n-1)^{n-3},
$$

hence for $X(1<x \leqq C)$ there exists $X_{1}\left(1<X_{1}<X\right)$ such that

$$
\begin{aligned}
f_{0}(X)= & \frac{n(2 n-1) B}{6(n-1)}(X-1)^{3}-\frac{n(n-2)(3 n-1) B}{12(n-1)^{2}}(X-1)^{4} \\
+ & \frac{(X-1)^{5}}{120} n(n-1)(n-2)(n-3)\left\{\left(n^{2}-1\right) X_{1}{ }^{2}-\left(10 n^{2}-9 n-4\right) X_{1}+3 n(7 n-8)\right\} \\
& \quad \times\left(n-X_{1}\right)^{n-6} .
\end{aligned}
$$

We have

$$
\left[\left(n^{2}-1\right) x^{2}-\left(10 n^{2}-9 n-4\right) x+3 n(7 n-8)\right]_{x=1}=3(n-1)(4 n-1)>0
$$

and

$$
\left(10 n^{2}-9 n-4\right) / 2\left(n^{2}-1\right)>2 \quad \text { for } n>3 / 2 \text {, }
$$

hence, assuming $C \leqq 2$, we obtain

$$
\begin{aligned}
& \left(n^{2}-1\right) X_{1}^{2}-\left(10 n^{2}-9 n-4\right) X_{1}+3 n(7 n-8) \\
\geqq & \left(n^{2}-1\right) C^{2}-\left(10 n^{2}-9 n-4\right) C+3 n(7 n-8):=q_{n} .
\end{aligned}
$$

Thus we obtain

$$
\begin{aligned}
f_{0}(X) & >\frac{n(2 n-1) B}{6(n-1)}(X-1)^{3}-\frac{n(n-2)(3 n-1) B}{12(n-1)^{2}}(X-1)^{4} \\
& +\frac{n(n-1)(n-2)(n-3) q_{n}\left(n-\varepsilon_{0}\right)^{n-6}}{120}(X-1)^{5} \quad \text { for } \quad 1<x \leqq C(\leqq 2),
\end{aligned}
$$

where $\varepsilon_{0}=1$ for $3 \leqq n<6$ and $\varepsilon_{0}=2$ for $n \geqq 6$.

Next, since we have

$$
\frac{d}{d X} \frac{X^{2}}{\sqrt{ } n-X}=\frac{X(4 n-3 X)}{2(n-X)^{3 / 2}} \text { and } \frac{d^{2}}{d X^{2}} \frac{X^{2}}{\sqrt{n-X}}=\frac{3 X^{2}-8 n X+8 n^{2}}{4(n-X)^{5 / 2}}>0,
$$

we obtain

$$
\frac{X^{2}}{\sqrt{n-X}}>\frac{1}{n-1}\left\{1+\frac{4 n-3}{2(n-1)}(X-1)\right\}
$$

Using (5.7) and (5.8), we obtain from (1.16) 


$$
\begin{aligned}
U_{5}(x)= & \frac{3 X^{2} f_{0}(X)}{(X-1)^{3} \sqrt{n-X}}>\frac{3}{\sqrt{n-1}}\left\{1+\frac{4 n-3}{2(n-1)}(X-1)\right\}\left\{\frac{n(2 n-1) B}{6(n-1)}\right. \\
& \left.-\frac{n(n-2)(3 n-1) B}{12(n-1)^{2}}(X-1)+\frac{n(n-1)(n-2)(n-3) q_{n}\left(n-\varepsilon_{0}\right)^{n-6}}{120}(X-1)^{2}\right\},
\end{aligned}
$$

which becomes the required one in the statement of the lemma. Q.E.D.

LEMMA 5.3. When $n>4$, we have

$$
\begin{aligned}
-U_{1}(x)> & -\frac{n(2 n-1)(n-1)^{n-5 / 2}}{2}+\frac{n\left(5 n^{2}-3 n+1\right)(n-1)^{n-7 / 2}}{4}(1-x) \\
+ & \left.\frac{n\left(8 n^{3}-50 n^{2}+44 n-9\right)(n-1)^{n-9 / 2}}{16}-\frac{3(n-2)(n-3)(7 n-8)(n-1)^{n-1 / 2} e_{n-1}}{40 n^{3}}\right\} \\
& \times(1-x)^{2}-\frac{n(n-2)(3 n-1)\left(8 n^{2}-8 n+3\right)(n-1)^{n-11 / 2}}{32}(1-x)^{3} \\
& \text { for } 0<x<1 .
\end{aligned}
$$

Proof. For $x(0<x<1)$ there exists $x_{1}\left(x<x_{1}<1\right)$ such that

$$
\begin{aligned}
f_{0}(x)= & -\frac{n(2 n-1) B}{6(n-1)}(1-x)^{3}-\frac{n(n-2)(3 n-1) B}{12(n-1)^{2}}(1-x)^{4} \\
- & \frac{(1-x)^{5}}{120} n(n-1)(n-2)(n-3)\left\{\left(n^{2}-1\right) x_{1}{ }^{2}-\left(10 n^{2}-9 n-4\right) x_{1}+3 n(7 n-8)\right\} \\
& \times\left(n-x_{1}\right)^{n-6} .
\end{aligned}
$$

We see easily that $f_{0}^{(6)}(x)<0$ for $0<x<1$, when $n>4$, and $f_{0}^{(5)}\left(x_{1}\right)<f_{0}^{(5)}(0)$ $=3 n^{n-4}(n-1)(n-2)(n-3)(7 n-8)$, which implies

$$
\begin{aligned}
f_{0}(x)> & -\frac{n(2 n-1) B}{6(n-1)}(1-x)^{3}-\frac{n(n-2)(3 n-1) B}{12(n-1)^{2}}(1-x)^{4} \\
& -\frac{(n-1)(n-2)(n-3)(7 n-8) n^{n-4}}{40}(1-x)^{5} .
\end{aligned}
$$

From (5.7) we obtain

$$
\frac{d^{3}}{d x^{3}} \frac{x^{2}}{\sqrt{n}-x}=\frac{3(x-2 n)(x-4 n)}{8(n-x)^{7 / 2}}>0 \quad \text { for } \quad 0<x<n .
$$

Using this and (5.7) with $x$ in place of $X$, we obtain

$$
\frac{x^{2}}{\sqrt{n-x}}<\frac{1}{\sqrt{n-1}}-\frac{4 n-3}{2(n-1)^{3 / 2}}(1-x)+\frac{8 n^{2}-8 n+3}{8(n-1)^{5 / 2}}(1-x)^{2} .
$$

Using (5.8) and (5.9), we obtain from (1.16) 


$$
\begin{aligned}
& -U_{1}(x)=\frac{3 x^{2} f_{0}(x)}{(1-x)^{3} \sqrt{n-x}}>-\frac{1}{\sqrt{n-1}}\left\{1-\frac{4 n-3}{2(n-1)}(1-x)+\frac{8 n^{2}-8 n+3}{8(n-1)^{2}}(1-x)^{2}\right\} \\
& \times\left\{\frac{n(2 n-1) B}{2(n-1)}+\frac{n(n-2)(3 n-1) B}{4(n-1)^{2}}(1-x)+\frac{3(n-1)(n-2)(n-3)(7 n-8) e_{n-1} B}{40 n^{3}}\right\}(1-x)^{2} \\
& =-\frac{n(2 n-1)(n-1)^{n-5 / 2}}{2}+\frac{n\left(5 n^{2}-3 n+1\right)(n-1)^{n-7 / 2}}{4}(1-x) \\
& +\left\{\frac{n\left(8 n^{3}-50 n^{2}+44 n-9\right)(n-1)^{n-9 / 2}}{16}-\frac{3(n-2)(n-3)(7 n-8)(n-1)^{n-1 / 2} e_{n-1}}{40 n^{3}}\right\}(1-x)^{2} \\
& -\frac{n(n-2)(3 n-1)\left(8 n^{2}-8 n+3\right)(n-1)^{n-11 / 2}}{32}(1-x)^{3} \\
& +\frac{3(n-2)(n-3)(7 n-8) e_{n-1}(n-1)^{n-5 / 2}}{320 n^{3}}-(1-x)^{3} \\
& \quad \times\left[4(n-1)(4 n-3)-\left(8 n^{2}-8 n+3\right)(1-x)\right],
\end{aligned}
$$

in which we see about the last part in the blackets that

$$
\begin{aligned}
& 4(n-1)(4 n-3)-\left(8 n^{2}-8 n+3\right)(1-x)>4(n-1)(4 n-3)-\cdots\left(8 n^{2}-8 n+3\right) \\
& =8 n^{2}-20+9>0 .
\end{aligned}
$$

Therefore, if we omit this part in the above expression, then we can obtain the required inequality.

Q.E.D.

We prepare here some formulas on $\psi(x)$. We have

$$
\left\{\begin{array}{l}
\phi(x)=x(n-x)^{n-1}, \quad \phi^{\prime}(x)=n(1-x)(n-x)^{n-2}, \\
\phi^{\prime \prime}(x)=-n(n-1)(2-x)(n-x)^{n-3}, \\
\phi^{\prime \prime \prime}(x)=n(n-1)(n-2)(3-x)(n-x)^{n-4}, \\
\psi^{(4)}(x)=-n(n-1)(n-2)(n-3)(4-x)(n-x)^{n-5}
\end{array}\right.
$$

LEMMA 5.4. When $n>3$, we have

$$
\begin{aligned}
U_{2}(x)>n^{2}(n-1)^{n-5 / 2} & -\frac{n^{2}(8 n-1)(n-1)^{n-7 / 2}}{6}(1-x) \\
& -\frac{n^{2}(n-2)(4 n-3)(n-1)^{n-9 / 2}}{3}(1-x)^{2} \quad \text { for } \quad 0<x<1 .
\end{aligned}
$$

Proof. For $x(0<x<1)$ there exists $x_{1}\left(x<x_{1}<1\right)$ such that

$$
B-x(n-x)^{n-1}=\frac{n(n-1)^{n-2}}{2}(1-x)^{2}+\frac{n(n-1)(n-2)\left(3-x_{1}\right)\left(n-x_{1}\right)^{n-4}}{6}(1-x)^{3},
$$

from which, assuming $n>3$, we obtain 


$$
\frac{B-x(n-x)^{n-1}}{(1-x)^{2}}>\frac{n(n-1)^{n-2}}{2}+\frac{n(n-2)(n-1)^{n-3}}{3}(1-x) \text {. }
$$

As in the proof of Lemma 5.2, we obtain

$$
\frac{x^{2}}{\sqrt{n-x}}>\frac{1}{\sqrt{n-1}}=\left\{1-\frac{4 n-3}{2(n-1)}(1-x)\right\} .
$$

Using (5.11) and (5.12), we obtain from (1.16)

$$
\begin{aligned}
U_{2}(x) & =\frac{2 n x^{2}\{B-\phi(x)\}}{(1-x)^{2} \sqrt{n-x}} \\
& >n^{2}(n-1)^{n-5 / 2}\left\{1-\frac{4 n-3}{2(n-1)}(1-x)\right\}\left\{1+\frac{2(n-2)}{3(n-1)}(1-x)\right\},
\end{aligned}
$$

which becomes the required one in the statement.

Q.E.D.

LEMMA 5.5. When $n>4$, we have

$$
\begin{aligned}
& -U_{6}(x)>-n^{2}(n-1)^{n-5 / 2}-\frac{n^{2}(8 n-1)(n-1)^{n-7 / 2}}{6}(X-1) \\
& +\left\{\frac{n^{2}(n-2)(13 n-3)(n-1)^{n-9 / 2}}{12}-\frac{n^{2}\left(8 n^{2}-8 n C+3 C^{2}\right)(n-1)^{n-2}}{8(n-C)^{5 / 2}}\right\}(X-1)^{2} \\
& -\frac{n^{2}(n-2)(n-3)(4 n-3)(n-1)^{n-11 / 2}}{8}(X-1)^{3} \quad \text { for } 1<X \leqq C \leqq \min \left(2, b_{n}\right) .
\end{aligned}
$$

Proof. For $X(1<X \leqq C)$ there exists $X_{1}\left(1<X_{1}<X\right)$ such that

$$
\begin{aligned}
B-X(n-X)^{n-1}= & \frac{n(n-1)^{n-2}}{2}(X-1)^{2}-\frac{n(n-2)(n-1)^{n-3}}{3}(X-1)^{3} \\
& +\frac{n(n-1)(n-2)(n-3)\left(4-X_{1}\right)\left(n-X_{1}\right)^{n-5}}{24}(X-1)^{4} .
\end{aligned}
$$

Since we have $\left((4-x)(n-x)^{n-5}\right)^{\prime}=-(n-4)(5-x)(n-x)^{n-6}<0$ for $n>4$ and $x<4$, we obtain easily

$$
\frac{B-X(n-X)^{n-1}}{(X-1)^{2}}<\frac{n(n-1)^{n-2}}{2}\left\{1-\frac{2(n-2)}{3(n-1)}(X-1)+\frac{(n-2)(n-3)}{4(n-1)^{2}}(X-1)^{2}\right\}
$$

Then there exists $X_{2}\left(1<X_{2}<X\right)$ such that

$$
\frac{X^{2}}{\sqrt{n-X}}=\frac{1}{\sqrt{n-1}}+\frac{4 n-3}{2(n-1)^{3 / 2}}(X-1)+\frac{3 X_{2}{ }^{2}-8 n X_{2}+8 n^{2}}{8\left(n-X_{2}\right)^{5 / 2}}(X-1)^{2}
$$

by means of (5.7). Since we have

we obtain

$$
\frac{d}{d X} \frac{3 X^{2}-8 n X+8 n^{2}}{(n-X)^{5 / 2}}=\frac{3\left(X^{2}-4 n X+8 n^{2}\right)}{2(n-X)^{7 / 2}}>0
$$




$$
\frac{X^{2}}{\sqrt{n-X}}<\frac{1}{\sqrt{n-1}}\left\{1+\frac{4 n-3}{2(n-1)}(X-1)+\frac{8 n^{2}-8 n C+3 C^{2}}{8(n-C)^{2}} \sqrt{\frac{n-1}{n-C}}(X-1)^{2}\right\} .
$$

Using (5.13) and (5.14), we obtain from (1.16)

$$
\begin{aligned}
& -U_{6}(x)=-\frac{2 n X^{2}\left\{B-\frac{\phi(X)\}}{(X-1)^{2}} \sqrt{n-X}\right.}{>-} n^{2}(n-1)^{n-5 / 2}\left\{1+\frac{4 n-3}{2(n-1)}(X-1)+\frac{8 n^{2}-8 n C+3 C^{2}}{8(n-C)^{2}} \sqrt{\frac{n-1}{n-C}}(X-1)^{2}\right\} \\
& \quad \times\left\{1-\frac{2(n-2)}{3(n-1)}(X-1)+\frac{(n-2)(n-3)}{4(n-1)^{2}}(X-1)^{2}\right\} \\
& =-n^{2}(n-1)^{n-5 / 2}-\frac{n^{2}(8 n-1)(n-1)^{n-7 / 2}}{6}(X-1) \\
& + \\
& \left.\quad-\frac{n^{2}(n-2)(13 n-3)(n-1)^{n-9 / 2}}{12}-\frac{n^{2}\left(8 n^{2}-8 n C+3 C^{2}\right)(n-1)^{n-2}}{8(n-C)^{5 / 2}}\right\}(X-1)^{2} \\
& \quad+n^{2}(n-1)^{n-5 / 2} \cdot \frac{8 n^{2}-8 n C+3 C^{2}}{8(n-C)^{2}} \sqrt{\frac{n-1}{n-C}} \cdot \frac{n-2}{n-1}\left[\frac{2}{3}-\frac{n-3}{4(n-1)}(X-1)\right](X-1)^{3},
\end{aligned}
$$

in which we see about the last part in the blackets that

$$
\frac{2}{3}-\frac{n-3}{4(n-1)}(X-1)>\frac{2}{3}-\frac{n-3}{4(n-1)}=\frac{5 n+1}{12(n-1)}>0 \text {. }
$$

Therefore, if we omit this part in the above expression, then we can obtain the required inequality.

Q.E.D.

LEMMA 5.6. When $n \geqq 4$, we have

$$
\begin{gathered}
U_{4}(x)>\frac{n^{2}\left(n^{2}-n+1\right)(n-1)^{n-7 / 2}}{6}(X-1)-\left\{\frac{n^{2}\left(2 n^{3}-4 n^{2}+6 n-3\right)(n-1)^{n-9 / 2}}{12}\right. \\
\left.+\frac{n^{2}(n-5)\left(n^{2}-n+1\right)(n-1)^{n-9 / 2}}{6}\left(\frac{n-\varepsilon_{4}}{n-1}\right)^{n-6}\right\}(X-1)^{2}+\frac{n^{2}(n-1)^{n-11 / 2}}{12} \\
\quad \times\left\{(n-5)\left(2 n^{3}-4 n^{2}+6 n-3\right)\left(\frac{n-\varepsilon_{4}}{n-1}\right)^{n-6}-\frac{(2 n-1)\left(4 n^{3}-7 n^{2}+9 n-4\right)}{2}\right\}(X-1)^{3} \\
+\frac{1}{24} n^{2}(2 n-1)(n-5)\left(4 n^{3}-7 n^{2}+9 n-4\right)(n-1)^{n-13 / 2}\left(\frac{n-\varepsilon_{4}}{n-1}\right)^{n-6}(X-1)^{4} \\
\quad \text { for } 1<X \leqq C \leqq 2,
\end{gathered}
$$

where we put $\varepsilon_{4}$ as follows: if $n \geqq 6, \varepsilon_{4}=1$; if $5<n<6, \varepsilon_{4}=C$; if $4 \leqq n \leqq 5, \varepsilon_{4}=-\infty$ and $C=1.225$ for $4 \leqq n<4.5$ and $C=1.163$ for $4.5 \leqq n \leqq 5$ as special cases.

Proof. By means of $(3.1) \sim(3.7)$, for $1<X \leqq C(\leqq 2)$ there exists $X_{1}\left(1<X_{1}<X\right)$ 
such that

$$
F_{2}(X)=\frac{(X-1)^{4}}{24} F_{2}{ }^{(4)}\left(X_{1}\right)=-\frac{n(n-1)}{24}(X-1)^{4}\left(n-X_{1}\right)^{n-5} \tilde{R}_{3}\left(X_{1}\right)
$$

On the other hand, from (3.7) we obtain

$$
\begin{aligned}
\tilde{R}_{3}^{\prime \prime}(x) & =6(n-1)(n+1)(n+2) x-2(n+1)\left(2 n^{3}+n^{2}+10 n-16\right) \\
& =2(n+1)\left\{3(n-1)(n+2) x-2 n^{3}-n^{2}-10 n+16\right\}
\end{aligned}
$$

and

$$
\begin{aligned}
& 3(n-1)(n+2) x-2 n^{3}-n^{2}-10 n+16 \leqq-\left(2 n^{3}-5 n^{2}+4 n-4\right) \\
& =-(n-2)\left(2 n^{2}-n+2\right)<0 \quad \text { for } 1 \leqq x \leqq 2 \text {, when } n>2 .
\end{aligned}
$$

Hence, $\tilde{R}_{3}(x)$ is convex upward in $1 \leqq x \leqq 2$. Since we have $\tilde{R}_{3}^{\prime}(1)>0$, we obtain

$$
\begin{aligned}
& \widetilde{R}_{3}\left(X_{1}\right)<\widetilde{R}_{3}(1)+\left(X_{1}-1\right) \tilde{R}_{3}^{\prime}(1)<\widetilde{R}_{3}(1)+(X-1) \widetilde{R}_{3}{ }^{\prime}(1) \\
& \quad=-4(n-1)^{2}\left(n^{2}-n+1\right)+2(n-1)\left(4 n^{3}-7 n^{2}+9 n-4\right)(X-1),
\end{aligned}
$$

which becomes $2(n-1)\left(2 n^{3}-3 n^{2}+5 n-2\right)>0$ at $X=2$ and $-(4 / 5)(n-1)\left(n^{3}-3 n+\right.$ $n-1)<0$ at $X=1.4$, respectively. We obtain

$$
F_{2}(X)>\frac{n(n-1)^{3}\left(n^{2}-n+1\right)}{6}(X-1)^{4}\left\{1-\frac{4 n^{3}-7 n^{2}+9 n-4}{2(n-1)\left(n^{2}-n+1\right)}(X-1)\right\}\left(n-X_{1}\right)^{n-5}
$$

There exists $X_{2}\left(1<X_{2}<X_{1}\right)$ such that

$$
\left(n-X_{1}\right)^{n-5}=(n-1)^{n-5}-(n-5)\left(n-X_{2}\right)^{n-6}\left(X_{1}-1\right),
$$

from which we obtain

$$
\left(n-X_{1}\right)^{n-5}>(n-1)^{n-5}\left\{1-\frac{n-5}{n-1}\left(\frac{n-\varepsilon_{4}}{n-1}\right)^{n-6}(X-1)\right\} \quad \text { for } \quad 1<X \leqq C,
$$

where if $n \geqq 6, \varepsilon_{4}=1$; if $5<n<6, \varepsilon_{4}=C$; if $4 \leqq n \leqq 5, \varepsilon_{4}=-\infty$.

Since we have

$$
\frac{d}{d x} \frac{x}{\sqrt{n-x}}=\frac{2 n-x}{2(n-x)^{3 / 2}}, \quad \frac{d^{2}}{d x^{2}} \frac{x}{\sqrt{n-x}}=\frac{4 n-x}{4(n-x)^{5 / 2}},
$$

we obtain easily

$$
\frac{X}{\sqrt{n-X}}>\frac{1}{\sqrt{n-1}}\left\{1+\frac{2 n-1}{2(n-1)}(X-1)\right\} \quad \text { for } \quad 1<X<n .
$$

Now using $(5.15) \sim(5.17)$ we obtain from (1.16) 


$$
\begin{aligned}
U_{4}(X)= & \frac{n X F_{2}(X)}{(n-1)(X-1)^{3} \sqrt{n-X}} \\
> & \frac{n^{2}\left(n^{2}-n+1\right)(n-1)^{n-7 / 2}}{6}(X-1)\left\{1-\frac{4 n^{3}-7 n^{2}+9 n-4}{2(n-1)\left(n^{2}-n+1\right)}(X-1)\right\} \\
& \quad \times\left\{1+\frac{2 n-1}{2(n-1)}(X-1)\right\}\left\{1-\frac{n-5}{n-1}\left(\frac{n-\varepsilon_{4}}{n-1}\right)^{n-6}(X-1)\right\} \\
= & \quad+\frac{n^{2}\left(n^{2}-n+1\right)(n-1)^{n-7 / 2}}{6}(X-1)-\left\{\frac{n^{2}\left(2 n^{3}-4 n^{2}+6 n-3\right)(n-1)^{n-9 / 2}}{12}\right. \\
& +\frac{n^{2}(n-1)^{n-11 / 2}}{12}\left\{(n-5)\left(2 n^{3}-4 n^{2}+6 n-3\right)\left(\frac{n-1)(n-1)^{n-9 / 2}}{n-1}\right)^{n-6}\left(\frac{n-\varepsilon_{4}}{n-1}\right)^{n-6}\right\}(X-1)^{2} \\
& \left.\quad-\frac{(2 n-1)\left(4 n^{3}-7 n^{2}+9 n-4\right)}{2}\right\}(X-1)^{3} \\
& +\frac{1}{24} n^{2}(2 n-1)(n-5)\left(4 n^{3}-7 n^{2}+9 n-4\right)(n-1)^{n-13 / 2}\left(\frac{n-\varepsilon_{4}}{n-1}\right)^{n-6}(X-1)^{4} .
\end{aligned}
$$

Finally, we shall give a main evaluation formula on $V\left(x, X_{n}(x)\right)$ near $x=1$. Using the inequalities in Lemmas $5.1 \sim 5.6$ in $V\left(x, X_{n}(x)\right)=-U_{1}(x)+U_{2}(x)+U_{5}(x)$ $-U_{6}(x)+U_{0}(x)+U_{4}(x)$, and noticing the part with $-U_{1}(x)+U_{2}(x)$ expressed a polynomial of $(1-x)$ :

$$
\begin{aligned}
& -\frac{n\left(n^{2}+7 n-3\right)(n-1)^{n-7 / 2}}{12}(1-x) \\
& -\left\{\frac{n\left(40 n^{3}-26 n^{2}-36 n+27\right)(n-1)^{n-9 / 2}}{48}\right. \\
& \left.\quad+\frac{3(n-2)(n-3)(7 n-8)(n-1)^{n-1 / 2} e_{n-1}}{40 n^{3}}\right\}(1-x)^{2} \\
& -\frac{n(n-2)(3 n-1)\left(8 n^{2}-8 n+3\right)(n-1)^{n-11 / 2}}{32}(1-x)^{3},
\end{aligned}
$$

whose coefficients of $(1-x),(1-x)^{2}$ and $(1-x)^{3}$ are always negative when $n \geqq 3$ and so becomes more smaller than it if we replace $1-x$ with $X-1$ since $X-1>1-x$ by Proposition 4 in (IV), and hence doing these replacements, we obtain the following

Proposition 4 . When $n>4$, we have

$$
V\left(x, X_{n}(x)\right)>\frac{n(2 n-1)\left(n^{2}-n-3\right)(n-1)^{n-7 / 2}}{6}(X-1)
$$




$$
\begin{aligned}
& -\left[\frac{n\left(8 n^{4}+44 n^{3}-108 n^{2}+102 n-9\right)}{48}+\frac{3 e_{n-1}(n-2)(n-3)(7 n-8)(n-1)^{4}}{40 n^{3}}\right. \\
& \quad+\frac{n^{2}\left(8 n^{2}-8 n C+3 C^{2}\right)}{8}\left(\frac{n-1}{n-C}\right)^{5 / 2}+\frac{n^{2}\left(n^{2}-n+1\right)\left\{(12 n-13) p_{n}+6 n-10\right\}}{36} \\
& \left.\quad+\frac{n^{2}(n-5)\left(n^{2}-n+1\right)}{6}\left(\frac{n-\varepsilon_{4}}{n-1}\right)^{n-6}\right](n-1)^{n-9 / 2}(X-1)^{2} \\
& +\left[-\frac{n\left(32 n^{5}+48 n^{4}-416 n^{3}+643 n^{2}-311 n+18\right)}{96}+\frac{n^{2}(4 n-5)\left(n^{2}-n+1\right) p_{n}\left(p_{n}+3 n-5\right)}{12}\right. \\
& \left.\quad+\frac{n^{2}(n-5)\left(2 n^{3}-4 n^{2}+6 n-3\right)}{12}\left(\frac{n-\varepsilon_{4}}{n-1}\right)^{n-6}\right](n-1)^{n-11 / 2}(X-1)^{3} \\
& +\left[\left[\left\{1+\frac{4 n-3}{2(n-1)}(X-1)\right\} \frac{n(n-2)(n-3)}{40} q_{n} \sqrt{n-1\left(n-\varepsilon_{0}\right)^{n-6}(X-1)^{2}}\right.\right. \\
& \left.\left.\quad+\frac{1}{24} n^{2}(2 n-1)(n-5)\left(4 n^{3}-7 n^{2}+9 n-4\right)(n-1)^{n-13 / 2}\left(\frac{n-\varepsilon_{4}}{n-1}\right)^{n-6}(X-1)^{4}\right]\right]
\end{aligned}
$$

where $C, p_{n}, q_{n}, \varepsilon_{0}$ and $\varepsilon_{4}$ are constants as in Lemmas $5.1 \sim 5.5$.

Remark. In the above expression, the quantity in the blackets $\mathbb{I} \mathbb{\text { is }}$ positive.

\section{§6. Proof of Theorem $\mathrm{C}$ for $6 \leqq n \leqq 9.7$.}

LEMMA 6.1. When $6 \leqq n \leqq 9.7$, we have

$$
\begin{array}{r}
V\left(x, X_{n}(x)\right)>\frac{n(n-1)^{n-11 / 2}}{6}(X-1)\left[(n-1)^{2}(2 n-1)\left(n^{2}-n-3\right)\right. \\
-\frac{1}{120}(n-1)\left(980 n^{4}+411 n^{3}-6052 n^{2}+9615 n-4457\right)(X-1) \\
\left.+\frac{1}{32}\left(124 n^{5}-807 n^{4}+1905 n^{3}-2315 n^{2}+1032 n-36\right)(X-1)^{2}\right] \\
\text { for } 1<X \leqq b_{n} .
\end{array}
$$

Proof. Omitting the last part in the blackets [ \of (5.18), we shall evaluate the coefficients of $(X-1)^{2}$ and $(X-1)^{3}$ of the right hand side. First, for $6 \leqq n \leqq 9.7$ we put $\varepsilon_{4}=1$ and $C=b_{n}\left(\leqq b_{6}=1.9117 \cdots\right)$ by Lemma 5.6 and have

$$
\begin{aligned}
& \frac{3 e_{n-1}(n-1)^{4}}{n^{3}}=3 n e_{n-1}\left(\frac{n-1}{n}\right)^{4} \leqq 3 n e_{8.7}\left(\frac{8.7}{9.7}\right)^{4}=3 n\left(\frac{9.7}{8.7}\right)^{4.7} \\
& =n \times 5.002739 \cdots,
\end{aligned}
$$




$$
\begin{aligned}
& \frac{8 n^{2}-8 n C+3 C^{2}}{8}\left(\frac{n-1}{n-C}\right)^{5 / 2}<\frac{8 n^{2}-16 n+12}{8}\left(\frac{n-1}{n-2}\right)^{5 / 2} \\
& \leqq \frac{2 n^{2}-4 n+3}{2}\left(\frac{5}{4}\right)^{5 / 2}=\left(2 n^{2}-4 n+3\right) \times 0.873464 \cdots<2 n^{2}-4 n+3,
\end{aligned}
$$

since $\left(8 n^{2}-8 n C+3 C^{2}\right) /(n-C)^{5 / 2}$ is increasing with respect to $C$, and

$$
\begin{aligned}
& (12 n-13) e_{n-2}+6 n-10 \leqq(12 n-13) e_{7.7}+6 n-10 \\
& \leqq(12 n-13) \times 2.5604698 \cdots+6 n-10 \\
& =36.725637 \cdots \times n-43.286107 \cdots<37 n-43 .
\end{aligned}
$$

Using these evaluations for the expressions, we obtain

$$
\begin{aligned}
& \frac{1}{48}\left(8 n^{4}+44 n^{3}-108 n^{2}+102 n-9\right)+\frac{1}{40}(n-2)(n-3)(7 n-8) \times 5.00274 \\
& \quad+n\left(2 n^{2}-4 n+3\right)+\frac{1}{36} n\left(n^{2}-n+1\right)(37 n-43)+\frac{n(n-5)\left(n^{2}-n+1\right)}{6} \\
& <\frac{1}{720}\left(980 n^{4}+411 n^{3}-6052 n^{2}+9615 n-4457\right),
\end{aligned}
$$

which is available for $6 \leqq n \leqq 9.7$.

Now, we proceed to the treatment of the terms regarding $(X-1)^{3}$ of $(5.18)$. We have for $6 \leqq n \leqq 9.7$

$$
\begin{aligned}
& e_{n-2}\left(e_{n-2}+3 n-5\right) \geqq e_{4}\left(e_{4}+3 n-5\right)=\left(\frac{5}{4}\right)^{8}+\left(\frac{5}{4}\right)^{4}(3 n-5) \\
& =5.96046 \cdots+(3 n-5) \times 2.44140 \cdots=7.32481 \cdots \times n-6.24657 \cdots \\
& >\frac{3(39 n-34)}{16}
\end{aligned}
$$

hence using this evaluation, we obtain

$$
\begin{aligned}
& -\frac{1}{96}\left(32 n^{5}+48 n^{4}-416 n^{8}+643 n^{2}-311 n+18\right) \\
& +\frac{1}{192} n(4 n-5)\left(n^{2}-n+1\right)(39 n-34)+\frac{1}{12} n(n-5)\left(2 n^{3}-4 n^{2}+6 n-3\right) \\
& =\frac{1}{192}\left(124 n^{5}-807 n^{4}+1905 n^{3}-2315 n^{2}+1032 n-36\right),
\end{aligned}
$$

which is available for $6 \leqq n \leqq 9.7$. 
Combining (6.1) and (6.2) with (5.18), we can obtain the inequality of this lemma.

Q.E.D.

Lemma 6.2. When $6 \leqq n \leqq 9.7$, we have

$$
V\left(x, X_{n}(x)\right)>0 \quad \text { for } \quad X_{n}{ }^{-1}\left(\frac{982-37 n}{882-37 n}\right) \leqq x<1 .
$$

This lemma will be supported by Lemma 6.1 and the following proposition which will be proved in another coming paper [18], because

$$
\frac{982-37 n}{882-37 n}<\frac{612}{512}=1.1953 \cdots<b_{9.7}=1.5882 \cdots .
$$

Proposition 5. When $6 \leqq n \leqq 9.7$, the quadratic polynomial of $t$

$$
\begin{aligned}
& (n-1)^{2}(2 n-1)\left(n^{2}-n-3\right)-\frac{t}{120}(n-1)\left(980 n^{4}+411 n^{3}-6052 n^{2}+9615 n-4457\right) \\
& +\frac{t^{2}}{32}\left(124 n^{5}-807 n^{4}+1905 n^{3}-2315 n^{2}+1032 n-36\right)>0 \\
& \text { for } 0 \leqq t \leqq \frac{100}{882-37 n} .
\end{aligned}
$$

On the other hand, according to Proposition 1 we cite here the approximately calculated values of $Y$ such that $K(n, Y)=0$ with $1<Y<n$ for each $n$ of Table 1.

Table 1.

\begin{tabular}{|r|c|c|c|c|c|c|c|c|c|c|c|}
\hline \multicolumn{1}{|c|}{$n$} & $Y$ & $Z$ & $n$ & $Y$ & $Z$ & $n$ & $Y$ & $Z$ & $n$ & $Y$ & $Z$ \\
\hline 6.0 & 1.3250 & 1.1515 & 7.0 & 1.3037 & 1.1605 & 8.0 & 1.2846 & 1.1706 & 9.0 & 1.2675 & 1.1820 \\
.1 & .3228 & .1523 & .1 & .3017 & .1614 & .1 & .2828 & .1717 & .1 & .2659 & .1833 \\
.2 & .3206 & .1532 & .2 & .2997 & .1624 & .2 & .2810 & .1728 & .2 & .2643 & .1846 \\
.3 & .3184 & .1541 & .3 & .2978 & .1634 & .3 & .2793 & .1739 & .3 & .2627 & .1859 \\
.4 & .3162 & .1549 & .4 & .2958 & .1644 & .4 & .2775 & .1750 & .4 & .2611 & .1871 \\
.5 & .3141 & .1558 & .5 & .2939 & .1654 & .5 & .2758 & .1762 & .5 & .2596 & .1885 \\
.6 & .3119 & .1567 & .6 & .2920 & .1664 & .6 & .2741 & .1773 & .6 & .2581 & .1898 \\
.7 & .3099 & .1577 & .7 & .2901 & .1674 & .7 & .2724 & .1785 & .7 & .2566 & .1911 \\
.8 & .3078 & .1586 & .8 & .2883 & .1685 & .8 & .2708 & .1797 & & & \\
.9 & .3057 & .1595 & .9 & .2864 & .1695 & .9 & .2691 & .1809 & $Y$ & $K(n, Y)=0$ \\
\hline
\end{tabular}

Now, noticing the values of $Y$ in Table 1 , we can prove that $K(n,(73-n) / 50)$ $>0$ for $6 \leqq n \leqq 9.7$ by Proposition 3 and an analogous argument used in the proof of Proposition 2 of (VI), where $(73-n) / 50$ is greater than $Y$ for the values of $n$ in the table. 
Next, we put $C=(73-n) / 50$ in (2.9) and obtain

$$
n-k_{n}\left(\frac{73-n}{50}\right)=\frac{(n+1)(23-n)+\sqrt{(n-1)\left(n^{3}-47 n^{2}+10775 n-15129\right)}}{2(73-n)}
$$

and we replace $y(n, X)$ defined by (3.8) in Proposition 1 with

$$
y=\left(\frac{n-X}{n-1}\right)^{n-k_{n}(C)}, \quad C=\frac{73-n}{50},
$$

and denote these functions by $K_{1}(n, X)$ and $K_{1}\left(n_{1}, n_{2} ; X_{1}, X_{2}\right)$. We cite here the approximately calculated values of $Y_{1}$ such that $K_{1}\left(n, Y_{1}\right)=0$ with $1<Y_{1}<n$ for the $n$ 's in Table 1. Table 2 tells us that

$$
Y_{1}<\frac{982-37 n}{882-37 n} \quad \text { for } \quad 7.6 \leqq n \leqq 9.7
$$

in the tables. We can also prove this inequality holds for any $n$ in this interval by the modified Proposition 3.

Table 2.

\begin{tabular}{|c|c|c|c|c|c|c|c|}
\hline$n$ & $Y_{1}$ & $n$ & $Y_{1}$ & $n$ & $Y_{1}$ & $n$ & $Y_{1}$ \\
\hline 6.0 & 1.2125 & 7.0 & 1.1814 & 8.0 & 1.1554 & 9.0 & 1.1334 \\
.1 & .2091 & .1 & .1786 & .1 & .1531 & .1 & .1314 \\
.2 & .2058 & .2 & .1759 & .2 & .1508 & .2 & .1294 \\
.3 & .2025 & .3 & .1731 & .3 & .1485 & .3 & .1274 \\
.4 & .1993 & .4 & .1705 & .4 & .1462 & .4 & .1255 \\
.5 & .1962 & .5 & .1679 & .5 & .1440 & .5 & .1236 \\
.6 & .1931 & .6 & .1653 & .6 & .1418 & .6 & .1217 \\
.7 & .1901 & .7 & .1628 & .7 & .1397 & .7 & .1199 \\
.8 & .1872 & .8 & .1603 & .8 & .1375 & \multicolumn{2}{|c|}{$K_{1}\left(n, Y_{1}\right)=0$} \\
.9 & .1843 & .9 & .1578 & .9 & .1355 & \\
\hline
\end{tabular}

Then, noticing the values $Y_{1}$ (for $6 \leqq n \leqq 7.6$ ), we can prove that

$$
K_{1}\left(n, \frac{37-n}{25}\right)>0 \quad \text { for } 6 \leqq n \leqq 7.6
$$

by the method described above. We put $C=(37-n) / 25$ in (2.9) and obtain

$$
n-k_{n}\left(\frac{37-n}{25}\right)=\frac{(n+1)(12-n)+\sqrt{(n-1)\left(n^{3}-25 n^{2}+2768 n-3844\right)}}{2(37-n)} .
$$

Next, we replace $y(n, X)$ in Proposition 1 with

$$
y=\left(\frac{n-X}{n-1}\right)^{n-k_{n}(C)}, \quad C=\frac{37-n}{25}
$$


and denote these functions by $K_{2}(n, X)$ and $K_{2}\left(n_{1}, n_{2} ; X_{1}, X_{2}\right)$. We cite here the approximately calculated values of $Y_{2}$ such that $K_{2}\left(n, Y_{2}\right)=0$ with $1<Y_{2}<n$ for the $n$ 's $(6 \leqq n \leqq 7.6)$ in Table 1 . Table 3 tells us that

$$
Y_{2}<\frac{982-37 n}{882-37 n} \quad \text { for } \quad 6.3 \leqq n \leqq 7.6
$$

in the tables. We can also prove this inequality for any $n$ in this interval by the modified Proposition 3.

Finally, we use $C$ in $k_{n}(C)$ as

$$
C=\frac{141-4 n}{100}
$$

and can prove

$$
K_{2}\left(n, \frac{141-4 n}{100}\right)>0 \quad \text { for } \quad 6 \leqq n \leqq 6.3
$$

Then we obtain

$$
n-k_{n}\left(\frac{141-4 n}{100}\right)=\frac{(n+1)(41-4 n)+\sqrt{(n-1)\left(16 n^{3}-344 n^{2}+43609 n-58081\right)}}{2(141-4 n)}
$$

and, using this function, compose $K_{3}(n, X)$ and $K_{3}\left(n_{1}, n_{2} ; X_{1}, X_{2}\right)$ by the same method above. And computing approximately the values $Y_{3}$ by $K_{3}\left(n, Y_{3}\right)=0$ for $6 \leqq n \leqq 6.3$, we obtain Table 4 , which shows

$$
Y_{3}<\frac{982-37 n}{882-37 n} \quad \text { for } 6 \leqq n \leqq 6.3 .
$$

Thus, we can conclude that

$$
V\left(x, X_{n}(x)\right)>0 \quad \text { for } \quad 0<x<1,
$$

when $6 \leqq n \leqq 9.7$, which assures Theorem $C$.

Table 3.

\begin{tabular}{|r|r|r|r|}
\hline$n$ & \multicolumn{1}{|c|}{$Y_{2}$} & $n$ & \multicolumn{1}{|c|}{$Y_{2}$} \\
\hline 6.0 & 1.1656 & 7.0 & 1.1250 \\
.1 & .1612 & .1 & .1213 \\
.2 & .1569 & .2 & .1177 \\
.3 & .1526 & .3 & .1141 \\
.4 & .1485 & .4 & .1106 \\
.5 & .1444 & .5 & .1071 \\
.6 & .1404 & .6 & .1037 \\
.7 & .1364 & \multicolumn{2}{|c|}{$K_{2}\left(n, Y_{2}\right)=0$} \\
.8 & .1326 & & \\
.9 & .1287 & & \\
\hline
\end{tabular}

Table 4.

\begin{tabular}{|c|c|}
\hline$n$ & $Y_{3}$ \\
\hline 6.0 & 1.1249 \\
.1 & .1205 \\
.2 & .1162 \\
.3 & .1120 \\
\hline
\end{tabular}


§7. Proof of Theorem $\mathrm{C}$ for $5 \leqq n \leqq 6$.

LEMMA 7.1. When $5 \leqq n \leqq 6$, for $1<X \leqq \min \left(2, b_{n}\right)$ we have

$$
\begin{aligned}
& V\left(x, X_{n}(x)\right)>\frac{n(n-1)^{n-11 / 2}}{6}(X-1)\left[(n-1)^{2}(2 n-1)\left(n^{2}-n-3\right)\right. \\
& -\frac{1}{120}(n-1)\left(1000 n^{4}+92 n^{3}-4864 n^{2}+7441 n-3245\right)(X-1) \\
& \left.+\frac{1}{32}\left(130 n^{5}-869 n^{4}+2027 n^{3}-2479 n^{2}+1108 n-36\right)(X-1)^{2}\right] \\
& \text { for } 1<X \leqq \min \left(2, b_{n}\right) .
\end{aligned}
$$

Proof. We shall evaluate the coefficients of $(X-1)^{2}$ and $(X-1)^{3}$ of the right hand side of (5.18). First, for $5 \leqq n \leqq 6$ we put $\varepsilon_{4}=C=\min \left(2, b_{n}\right)$ by Lemma 5.1 and have

$$
\begin{aligned}
& \frac{3 e_{n-1}(n-1)^{4}}{n^{8}}=3 n e_{n-1}\left(\frac{n-1}{n}\right)^{4} \leqq 3 n e_{5}\left(\frac{5}{6}\right)^{4}=\frac{18}{5} n=3.6 n \\
& \frac{8 n^{2}-8 n C+3 C^{2}}{8}\left(\frac{n-1}{n-C}\right)^{5 / 2} \leqq \frac{8 n^{2}-16 n+12}{8}\left(\frac{n-1}{n-2}\right)^{5 / 2} \leqq \frac{2 n^{2}-4 n+3}{2}\left(\frac{4}{3}\right)^{5 / 2} \\
& \quad=\left(2 n^{2}-4 n+3\right) \times 1.0264004 \cdots<\left(2 n^{2}-4 n+3\right) \times 1.0265, \\
& (12 n-13) e_{n-2}+6 n-10 \leqq(12 n-13) e_{4}+6 n-10 \\
& \quad=(12 n-13) \times \frac{625}{256}+6 n-10=\frac{9036 n-10685}{256}<36 n-41
\end{aligned}
$$

and

$$
\left(\frac{n-\varepsilon_{4}}{n-1}\right)^{n-6}=\left(\frac{n-1}{n-C}\right)^{6-n} \leqq\left(\frac{n-1}{n-2}\right)^{6-n} \leqq\left(\frac{4}{3}\right)^{6-n} \leqq \frac{4}{3} .
$$

Using these evaluations for the expressions in the blackets corresponding to $(X-1)^{2}$ of $(5.18)$, we obtain

$$
\begin{aligned}
& \frac{1}{48}\left(8 n^{4}+44 n^{3}-108 n^{2}+102 n-9\right)+\frac{1}{40}(n-2)(n-3)(7 n-8) \times 3.6 \\
& \quad+n\left(2 n^{2}-4 n+3\right) \times 1.0265+\frac{1}{36} n\left(n^{2}-n+1\right)(36 n-41)+\frac{2}{9} n(n-5)\left(n^{2}-n+1\right) \\
& <\frac{1}{720}\left(1000 n^{4}+92 n^{3}-4864 n^{2}+7441 n-3245\right)
\end{aligned}
$$

Next, we proceed to the treatment of the terms regarding $(X-1)^{3}$. We have for $5 \leqq n \leqq 6$

$$
e_{n-2}\left(e_{n-2}+3 n-5\right) \geqq e_{3}\left(e_{3}+3 n-5\right)=\frac{4^{3}}{3^{6}}(81 n-71) .
$$


Using this evaluation, we obtain

$$
\begin{aligned}
- & \frac{1}{96}\left(32 n^{5}+48 n^{4}-416 n^{3}+643 n^{2}-311 n+18\right) \\
& +\frac{1}{36} \cdot \frac{4^{3}}{3^{6}} n(4 n-5)\left(n^{2}-n+1\right)(81 n-71)+\frac{1}{12} \cdot \frac{4}{3} n(n-5)\left(2 n^{3}-4 n^{2}+6 n-3\right) \\
= & \frac{1}{2^{5} \cdot 3^{8}}\left(142560 n^{5}-950244 n^{4}+2216736 n^{3}-2710593 n^{2}+1211837 n-39366\right) \\
> & \frac{1}{192}\left(130 n^{5}-869 n^{4}+2027 n^{3}-2479 n^{2}+1108 n-36\right) .
\end{aligned}
$$

Combining (7.1) and (7.2) with (5.18), we can obtain the inequality of this lemma. Q.E.D.

LEMMA 7.2. When $5 \leqq n \leqq 6$, we have

$$
V\left(x, X_{n}(x)\right)>0 \quad \text { for } \quad X_{n}^{-1}\left(\frac{57-3 n}{52-3 n}\right) \leqq x<1 .
$$

This lemma will be supported by Lemma 7.1 and the following proposition which will be proved also in [18], because

$$
\frac{57-3 n}{52-3 n} \leqq \frac{39}{34}=1.14770 \cdots<b_{6}=1.9117 \cdots \leqq b_{n} \quad \text { for } \quad 5 \leqq n \leqq 6 .
$$

PROPOSITION 6 . When $5 \leqq n \leqq 6$, the quadratic polynomial of $t$.

$$
\begin{aligned}
& (n-1)^{2}(2 n-1)\left(n^{2}-n-3\right)-\frac{t}{120}(n-1)\left(1000 n^{4}+92 n^{3}-4864 n^{2}+7441 n-3245\right) \\
& +\frac{t^{2}}{32}\left(130 n^{5}-869 n^{4}+2027 n^{3}-2479 n^{2}+1108 n-36\right)>0 \\
& \text { for } 0 \leqq t \leqq \frac{5}{52-3 n} .
\end{aligned}
$$

Now, we cite here the approximately calculated values of $Y$ analogous to Table 1 for $5 \leqq n \leqq 6$, in Table $1^{\prime}$, in which $Z_{1}=(57-3 n) /(52-3 n)$. Noticing the values of $Y$ and we can also prove

$$
K\left(n, \frac{73-n}{50}\right)>0 \quad \text { for } \quad 5 \leqq n \leqq 6 .
$$

Using the revised $K_{1}(n, X)$ as in $\S 6$, we cite the approximately calculated values of $Y_{\mathrm{I}}$ analogous to Table 2 in Table $2^{\prime}$.

Noticing the values of $Y_{1}$ in Table $2^{\prime}$, we can also prove that

$$
K_{1}\left(n, \frac{37-n}{25}\right)>0 \quad \text { for } 5 \leqq n \leqq 6 .
$$

Then, using $K_{2}(n, X)$ as in $\S 6$, we cite the approximately calculated values of $Y_{2}$ analogous to Table 3 . 
Table 1'.

\begin{tabular}{|r|c|c|}
\hline$n$ & $Y$ & $Z_{1}$ \\
\hline 5.0 & 1.3492 & 1.1351 \\
.1 & .3466 & .1362 \\
.2 & .3441 & .1373 \\
.3 & .3416 & .1385 \\
.4 & .3391 & .1396 \\
.5 & .3367 & .1408 \\
.6 & .3343 & .1420 \\
.7 & .3319 & .1432 \\
.8 & .3296 & .1445 \\
.9 & .3273 & .1457 \\
6.0 & 1.3250 & 1.1470 \\
\hline
\end{tabular}

Table $2^{\prime}$.

\begin{tabular}{|r|r|}
\hline$n$ & $Y_{1}$ \\
\hline 5.0 & 1.2507 \\
.1 & .2406 \\
.2 & .2423 \\
.3 & .2383 \\
.4 & .2344 \\
.5 & .2305 \\
.6 & .2268 \\
.7 & .2231 \\
.8 & .2195 \\
.9 & .2159 \\
6.0 & 1.2125 \\
\hline
\end{tabular}

Noticing Table $3^{\prime}$, we can prove

$$
K_{2}\left(n, \frac{76-3 n}{50}\right)>0 \quad \text { for } \quad 5 \leqq n \leqq 5.5
$$

and

$$
K_{2}\left(n, \frac{141-4 n}{100}\right)>0 \quad \text { for } \quad 5.5 \leqq n \leqq 6 .
$$

Then, using $K_{3}(n, X)$ for $5.5 \leqq n \leqq 6$, we obtain the approximately calculated values $Y_{3}$ as in $\S 6$. Here we cite them in Table $4^{\prime}$, which shows

$$
Y_{3}<\frac{57-3 n}{52-3 n}=Z_{1} \quad \text { for } \quad 5.7 \leqq n \leqq 6 .
$$

For $5 \leqq n \leqq 5.5$, we use

$$
k_{n}(C)=k_{n}\left(\frac{76-3 n}{50}\right)
$$

Then, we have

$$
n-k_{n}(C)=\frac{(n+1)(26-3 n)+\sqrt{(n-1)\left(9 n^{3}-165 n^{2}+11432 n-15876\right)}}{2(76-3 n)},
$$

and, using this function, compose $K_{4}(n, X)$ and $K_{4}\left(n_{1}, n_{2} ; X_{1}, X_{2}\right)$ by the same method as in $\S 6$. And computing approximately the values of $Y_{4}$ by $K_{4}\left(n, Y_{4}\right)$ $=0$ for $5 \leqq n \leqq 5.5$ as in Table $4^{\prime}$.

Noticing Table 4 ', we can prove

$$
K_{3}\left(n, \frac{189-8 n}{125}\right)>0 \quad \text { for } \quad 5.5 \leqq n \leqq 6,
$$


Table $3^{\prime}$.

\begin{tabular}{|r|r|r|r|}
\hline$n$ & \multicolumn{1}{|c|}{$Y_{2}$} & $n$ & \multicolumn{1}{|c|}{$Y_{2}$} \\
\hline 5.0 & 1.2143 & 5.6 & 1.1839 \\
.1 & .2090 & .7 & .1792 \\
.2 & .2038 & .8 & .1746 \\
.3 & .1987 & .9 & .1700 \\
.4 & .1937 & 6.0 & 1.1656 \\
.5 & .1888 & & \\
\hline
\end{tabular}

Table $4^{\prime}$.

\begin{tabular}{|c|c|c|c|}
\hline$n$ & $Y_{4}$ & $n$ & \multicolumn{1}{|c|}{$Y_{3}$} \\
\hline 5.0 & 1.1806 & 5.5 & 1.1482 \\
.1 & .1739 & .6 & .1434 \\
.2 & .1673 & .7 & .1386 \\
.3 & .1609 & .8 & .1339 \\
.4 & .1545 & .9 & .1293 \\
.5 & .1482 & 6.0 & .1249 \\
\hline
\end{tabular}

$$
K_{4}\left(n, \frac{189-8 n}{125}\right)>0 \quad \text { for } \quad 5 \leqq n \leqq 5.5 .
$$

Next, for $5 \leqq n \leqq 5.7$, we use

$$
k_{n}(C)=k_{n}\left(\frac{189-8 n}{125}\right)
$$

Then we obtain

$$
n-k_{n}(C)=\frac{4(n+1)(8-n)+\sqrt{(n-1)\left(16 n^{3}-272 n^{2}+17905 n-24649\right)}}{189-8 n}
$$

and, using this function, compose $K_{5}(n, X)$ and $K_{5}\left(n_{1}, n_{2} ; X_{1}, X_{2}\right)$. Then computing approximately the values of $Y_{5}$ by $K_{5}\left(n, Y_{5}\right)=0$ for $5 \leqq n \leqq 5.7$. We cite here the values $Y_{5}$ in Table 5 , which shows

$$
Y_{5}<\frac{57-3 n}{52-3 n}=Z_{1} \quad \text { for } \quad 5.4 \leqq n \leqq 5.7 .
$$

For $5 \leqq n \leqq 5.4$, we can prove

$$
K_{5}\left(n, \frac{1503--68 n}{1000}\right)>0
$$

by means of $K_{5}\left(n_{1}, n_{2} ; X_{1}, X_{2}\right)$ as the previous case.

Then, taking $C=(1503-68 n) / 1000$, we obtain

$$
n-k_{n}(C)=\frac{(n+1)(503-68 n)+\sqrt{(n-1)\left(4624 n^{3}-73032 n^{2}+4593417 n-6265009\right)}}{2(1503-68 n)}
$$

and, using this as the exponent of $y(n, X)$, compose $K_{6}(n, X)$ and $K_{6}\left(n_{1}, n_{2} ; X_{1}, X_{2}\right)$ as the previous cases. Computing approximately the values of $Y_{6}$ by $K_{6}\left(n, Y_{6}\right)=0$ for $5 \leqq n \leqq 5.4$, we obtain Table 6 , which shows

$$
Y_{6}<\frac{57-3 n}{52-3 n}=Z_{1} \quad \text { for } \quad 5.1 \leqq n \leqq 5.4 .
$$

For $5 \leqq n \leqq 5.1$, we can prove 
Table 5.

\begin{tabular}{|r|r|r|r|}
\hline$n$ & \multicolumn{1}{|c|}{$Y_{5}$} & $n$ & \multicolumn{1}{|c|}{$Y_{5}$} \\
\hline 5.0 & 1.1628 & 5.4 & 1.1351 \\
.1 & .1556 & .5 & .1285 \\
.2 & .1487 & .6 & .1220 \\
.3 & .1418 & .7 & .1156 \\
\hline
\end{tabular}

Table 6.

\begin{tabular}{|c|c|}
\hline$n$ & $Y_{6}$ \\
\hline 5.0 & 1.1427 \\
.1 & .1351 \\
.2 & .1278 \\
.3 & .1205 \\
.4 & .1134 \\
\hline
\end{tabular}

$$
K_{6}\left(n, \frac{1544-80 n}{1000}\right)>0
$$

Table 7.

\begin{tabular}{|c|c|}
\hline$n$ & $Y_{7}$ \\
\hline 5.0 & 1.1286 \\
.1 & .1201 \\
\hline
\end{tabular}

by means of $K_{6}\left(n_{1}, n_{2} ; X_{1}, X_{2}\right)$ as the previous cases.

Finally, for $5 \leqq n \leqq 5.1$, taking

$$
C=\frac{1544-80 n}{1000}=\frac{193-10 n}{125},
$$

we obtain

$$
n-k_{n}(C)=\frac{(n+1)(68-10 n)+\sqrt{(n-1)\left(100 n^{3}-1460 n^{2}+73484 n-101124\right)}}{2(193-10 n)}
$$

and, using this as the exponent of $y(n, X)$, compose $K_{7}(n, X)$ and $K_{7}\left(n_{1}, n_{2} ; X_{1}, X_{2}\right)$ as the previous cases. Computing approximately the values of $Y_{7}$ by $K_{7}\left(n, Y_{7}\right)=0$ for $5 \leqq n \leqq 5.1$, we obtain Table 7 , which shows

$$
Y_{7}<\frac{57-3 n}{52-3 n}=Z_{1} \quad \text { for } \quad 5 \leqq n \leqq 5.1 .
$$

Thus we can conclude that

$$
V\left(x, X_{n}(x)\right)>0 \quad \text { for } 0<x<1 \text {, }
$$

when $5 \leqq n \leqq 6$, which implies Theorem $C$ for $5 \leqq n \leqq 6$.

We have obtained the main theorem of this paper as follows.

THEOREM C. The period function $T$ as function of $\tau$ and $n$ is monotone decreasing with respect to $n \geqq 5$ for any fixed $\tau(0<\tau<1)$.

Remark. When we composed $K_{\imath}\left(n_{1}, n_{2} ; X_{1}, X_{2}\right)$, we used the following inequalities in place of (4.13) which can be proved by Lemma 2.3, supposing $C=C(n)$ is a decreasing function of $n$ :

$$
\left(\frac{n_{1}-X_{2}}{n_{1}-1}\right)^{n_{2}-k_{n_{2}}\left(C\left(n_{2}\right)\right)} \leqq\left(\frac{n-X}{n-1}\right)^{n-k_{n}(C(n))} \leqq\left(\frac{n_{2}-X_{1}}{n_{2}-1}\right)^{n_{1}-k_{n_{1}}\left(C\left(n_{1}\right)\right)} .
$$

\section{REFERENCES}

[1] S. S. Chern, M. do Carmo and S. Kobayashi, Minimal Submanifolds of a sphere with second fundamental form of constant length, Functional Analysis and Related Fields, Springer-Verlag, 1970, 60-75. 
[2] S. FuRUYA, On periods of periodic solutions of a certain nonlinear differential equation, Japan-United States Seminar on Ordinary Differential and Functional Equations, Lecture Notes in Mathematics, Springer-Verlag, 243 (1971), 320-323.

[3] W. Y. Hsiang and H. B. Lawson, Jr., Minimal submanifolds of low cohomogeneity, J. Diff. Geometry, 5 (1970), 1-38.

[4] T. OTsukI, Minimal hypersurfaces in a Riemannian manifold of constant curvature, Amer. J. Math., 92 (1970), 145-173.

[5] T. Oтsuki, On integral inequalities related with a certain nonlinear differential equation, Proc. Japan Acad., 48 (1972), 9-12.

[6] T. Otsuki, On a 2-dimensional Riemannian manifold, Differential Geometry, in honor of K. Yano, Kinokuniya, Tokyo, 1972, 401-414.

[7] T. Otsuki, On a family of Riemannian manifolds defined on an $m$-disk, Math. J. Okayama Univ., 16 (1973), 85-97.

[8] T. OTsuks, On a bound for periods of solutions of a certain nonlinear differential equation (I), J. Math. Soc. Japan, 26 (1974), 206-233.

[9] T. Oтsuki, On a bound for periods of solutions of a certain nonlinear differential equation (II), Funkcialaj Ekvacioj. 17 (1974), 193-205.

[10] T. OTsuk, Geodesics of $O_{n}^{2}$ and an analysis on a related Riemann surface, Tôhoku Math. J., 28 (1976), 411-427.

[11] T. Otsukr, A certain property of geodesics of the family of Riemannian manifolds $O_{n}^{2}$ (I), Proc. of Japan-United States Seminar on Minimal Submanifolds, including Geodesics, Kaigai, Tokyo, 1978, 173-192.

[12] T. OTsukI, A certain property of geodesics of the family of Riemannian manifolds $O_{n}^{2}$ (II), Kodai Math. J., 2 (1979), 211-242.

[13] T. OTsuki, A certain property of geodesics of the family of Riemannian manifolds $O_{n}^{2}$ (III), Kodai Math. J., 4 (1981), 28-70.

[14] T. Otsuki, A certain property of geodesics of the family of Riemannian manifolds $O_{n}^{2}$ (IV). Kodai Math. J., 5 (1982), 160-199.

[15] T. OTsukI, A certain property of geodesics of the family of Riemannian manifolds $O_{n}^{2}(\mathrm{~V})$, Kodai Math. J., 5 (1982), 454-481.

[16] T. Otsuki, A certain property of geodesics of the family of Riemannian manifolds $O_{\bar{n}}^{2}$ (VI), Kodai Math. J., 7 (1984), 326-364.

[17] T. OTsukI, Certain inequalities related with a non-linear differential equation (I), (II), TRU Math., 20 (1984), 23-68, 69-89.

[18] T. OTsukI, Certain inequalities and constants related with a non-linear differential equation, TRU Math., 21 (1985), 5-35.

[19] T. OTsukı, A family of 2-dimensional Riemannian manifolds joining the noneuclidean planes, TRU Math., 17 (1981), 129-139.

[20] M. Maeda and T. OTsuki, Models of the Riemannian manifolds $O_{n}^{2}$ in the Lorentzian 4-space, J. Diff. Geometry, 9 (1974), 97-108.

[21] M. URABE, Computations of periods of a certain nonlinear autonomous oscilations, Study of algorithms of numerical computations, Sūrikaiseki Kenkyūsho Kōkyūroku, 149 (1972), 111-129 (Japanese).

Department of Mathematics

SCIENCE UNIVERSITY OF TOKYO

WaKamiya-cho 26, ShinjukU-Ku

TOKYO, JAPAN 162 